\title{
Primordial power spectrum from Planck
}

\section{Dhiraj Kumar Hazra ${ }^{a}$ Arman Shafieloo $^{a, b}$ Tarun Souradeep ${ }^{c}$}

\author{
${ }^{a}$ Asia Pacific Center for Theoretical Physics, Pohang, Gyeongbuk 790-784, Korea \\ ${ }^{b}$ Department of Physics, POSTECH, Pohang, Gyeongbuk 790-784, Korea \\ ${ }^{c}$ Inter-University Centre for Astronomy and Astrophysics, Post Bag 4, Ganeshkhind, \\ Pune 411 007, India \\ E-mail: dhiraj@apctp.org, arman@apctp.org, tarun@iucaa.ernet.in
}

\begin{abstract}
Using modified Richardson-Lucy algorithm we reconstruct the primordial power spectrum (PPS) from Planck Cosmic Microwave Background (CMB) temperature anisotropy data. In our analysis we use different combinations of angular power spectra from Planck to reconstruct the shape of the primordial power spectrum and locate possible features. Performing an extensive error analysis we found the dip near $\ell \sim 750-850$ represents the most prominent feature in the data. Feature near $\ell \sim 1800-2000$ is detectable with high confidence only in $217 \mathrm{GHz}$ spectrum and is apparently consequence of a small systematic as described in the revised Planck 2013 papers. Fixing the background cosmological parameters and the foreground nuisance parameters to their best fit baseline values, we report that the best fit power law primordial power spectrum is consistent with the reconstructed form of the PPS at $2 \sigma$ C.L. of the estimated errors (apart from the local features mentioned above). As a consistency test, we found the reconstructed primordial power spectrum from Planck temperature data can also substantially improve the fit to WMAP-9 angular power spectrum data (with respect to power-law form of the PPS) allowing an overall amplitude shift of $\sim 2.5 \%$. In this context low- $\ell$ and $100 \mathrm{GHz}$ spectrum from Planck which have proper overlap in the multipole range with WMAP data found to be completely consistent with WMAP-9 (allowing amplitude shift). As another important result of our analysis we do report the evidence of gravitational lensing through the reconstruction analysis. Finally we present two smooth form of the PPS containing only the important features. These smooth forms of PPS can provide significant improvements in fitting the data (with respect to the power law PPS) and can be helpful to give hints for inflationary model building.
\end{abstract}




\section{Contents}

1 Introduction and the road-map 1

2 Reconstruction algorithm $\quad 3$

3 Applications and results $\quad 7$

$\begin{array}{lll}3.1 & \text { Consistency with WMAP data } & 7\end{array}$

$\begin{array}{lll}3.2 & \text { Reconstruction indicates lensing } & 12\end{array}$

3.3 Features: Where are they? 16

3.4 Error estimation : Hunting down the probable physical features 20

3.5 Filtering out the noise: towards a smooth primordial power spectrum 23

\section{Introduction and the road-map}

Planck [1], the most recent full sky Cosmic Microwave Background (CMB) survey has been able to provide us with the most precise information about the early Universe. However, few underlying assumptions about the Universe largely dominate our understanding of the history of the Universe. The angular power spectrum from CMB temperature fluctuations contains a convolved signal of the shape of initial fluctuation, i.e. the primordial power spectrum (PPS) and the transition of this fluctuation through different phases of the Universe. To extract these information we usually make assumptions in two sectors, namely the formation and evolution of the perturbations/fluctuations. The first assumption we make in primordial formation sector where the primordial power spectrum is assumed to be nearly scale invariant, following the theory of slow-roll inflation. In the evolution sector, we model the evolution of the initial perturbations.

Favored deviations from our standard assumptions may hint towards the necessity of a new model of the Universe. Hunting down these deviations requires extensive search in both the sectors mentioned, individually or jointly. The nature of the search can be model specific or model independent. Model specific search, though being effective, is limited in terms of its flexibilities. On the other hand, model independent reconstruction of the phenomenology directly from the data can immediately pin point all the places where we might need deviations.

The aim of this paper is to reconstruct the PPS from Planck data. We list few issues that can be addressed with the PPS reconstruction.

- Features : Features in the PPS [2] have been widely discussed in literature to address the deviations from power law PPS and thereby categorize a class of inflationary models that deal with departures from slow-roll hypothesis. The location of possible features can be addressed by direct reconstruction that is one 
of the main aims of this paper. Moreover, Planck had observed the CMB in 9 different frequency channels and provided 5 angular power spectra for parameter estimation. It is interesting to examine whether features found in different spectra are consistent to each other.

- Falsifying power law PPS : Certain deviations from power law PPS can address the data better with additional degrees of freedom. However it does not always mean that these features indicate any physical effect. Statistical fluctuations and noise or systematics in the data can also lead to certain features. Reconstruction of PPS for a large number of realizations of the data can address this issue and in our work [3] with WMAP-9 data [4] we have shown that within the uncertainties in the WMAP data the power law performs perfectly well. Planck constrains the PPS with much better precision than WMAP-9 which invites a re-analysis of the falsification of power law PPS with Planck data. Moreover, Planck analysis has reported features near multipoles $\ell \simeq 20-30$ and near $\ell \simeq 1800^{*}$. In our analysis we provide the significance of these features in different spectra provided by Planck.

- Gravitational lensing : With the Planck data the effect of gravitational lensing is confirmed with $25 \sigma$ confidence [6]. The convolved information of the initial fluctuations and its evolution reach us after getting lensed by matter distributed across the Universe. Due to this convolution we can expect a degeneracy between the background cosmology, the gravitational lensing and the shape of the PPS. In this paper we address the degeneracy between the effects of gravitational lensing and the PPS in detail. Despite of the degeneracy it is interesting to examine whether the lensing effect can be captured/indicated through the reconstruction.

- Consistency with WMAP-9: Resolution of Planck is significantly better than WMAP and hence cosmological scales probed by Planck includes WMAP probed scales. This overlap enables us to check how well the reconstructed PPS from Planck can fit the WMAP dataset. We explore the consistency in PPS obtained from different combinations of angular power spectra provided by Planck.

- Smooth primordial power spectrum : Having investigating all the above issues, we present a smooth PPS which can be described by a simple form. We expect the smooth PPS to contain only significant features in the data, which might be coming from some underlying physical effects.

This paper is organized as follows. In section 2 we discuss the main algorithm of the reconstruction. Following that, in section 3 we present the results of our analysis and in section 4 we end with concluding remarks.

\footnotetext{
$* \ell=1800$ feature is acknowledged by the Planck collaboration to be a systematic caused by $4 \mathrm{~K}$ cooler-bolometer read-out electromagnetic interference [5].
} 


\section{Reconstruction algorithm}

Reconstruction of the PPS directly from the data can be achieved through various methods $[3,7-11]^{\dagger}$. In this analysis we shall re-use the Richardson-Lucy (RL) [13-16] deconvolution algorithm. The error-sensitive IRL method was introduced in $[9,10]$ for the binned data. We further modified the IRL algorithm to work for WMAP-9 in case of binned and unbinned data in a combined analysis [3], referred to as MRL. However, data from Planck necessitates yet another modification to the MRL algorithm.

Let us revisit the convolution (Eq. 2.1) of PPS $\left(P_{k}\right)$ and the radiative transport kernel $\left(G_{\ell k}\right)$ that generates the angular power spectrum $\left(\mathcal{C}_{\ell}^{\mathrm{T}}\right)$.

$$
\mathcal{C}_{\ell}^{\mathrm{T}}=\sum_{i} G_{\ell k_{i}} P_{k_{i}}
$$

The radiative transport kernel here contains the information about the assumed background cosmological model. We should mention that throughout this paper we have used the Friedmann-Lemaître-Robertson-Walker spatially flat Universe with cosmological constant as the dark energy. The dark matter is assumed to be nonrelativistic (CDM). Following the standard model the effective number of relativistic species is fixed to be 3.046. For neutrinos we follow standard mass hierarchy and mass of the single massive neutrino eigenstate is fixed to be $m_{\nu}=0.06 \mathrm{eV}$. Note our assumptions simply follow the baseline model of the Planck analysis [17]. The radiative transport matrix $G_{\ell k}$ is calculated for the Planck best fit values of the parameters $\Omega_{\mathrm{b}} h^{2}$ (baryon density), $\Omega_{\mathrm{CDM}} h^{2}$ (CDM density), $H_{0}$ (Hubble parameter) and $\tau$ (reionization optical depth).

It is of importance that we present the form of the radiative transport kernel pictorially. The angular power spectrum at multipole $\ell$ is related to a window of the PPS in wavenumber $(k)$ space. The particular window in $k$ space is given by the distribution of radiative transport kernel which is plotted in Fig. 1. In this figure we have plotted the normalized $G_{\ell k}$ (maximum value normalized to 1 ) as a function of $k$. The colorbar at the bottom represents the multipole from $\ell=50$ to 2500 . In the inset the same is plotted for low- $\ell(2-49)$, in logarithmic scale in $k$. Note that the peak-position of the kernel (corresponding to a particular color) refers to the $k$ that contributes maximum to the convolution integral of angular power spectrum at a particular $\ell$ (corresponding to the same color).

The $\mathcal{C}_{\ell}^{\mathrm{T}}$ appearing in Eq. 2.1 does not exactly correspond to the angular power spectrum from $\mathrm{CMB}$ map that we measure. The signal $\mathcal{C}_{\ell}^{\mathrm{T}}$ gets lensed by the underlying matter distribution while reaching to us. Moreover, we should remember that at small scales due to point sources, cosmic infrared background (CIB), Sunyaev-Zeldovich (SZ) effect the foregrounds dominate the power spectrum. Hence, to reconstruct the PPS from the angular power spectrum obtained from Planck we need to extract the $\mathcal{C}_{\ell}^{\mathrm{T}}$ information of Eq. 2.1 from the $\mathcal{C}_{\ell}^{\text {Data }}$ in all Planck spectra.

\footnotetext{
${ }^{\dagger}$ Also see [12], though we do not agree with some claims made in this paper regarding the MRL algorithm.
} 


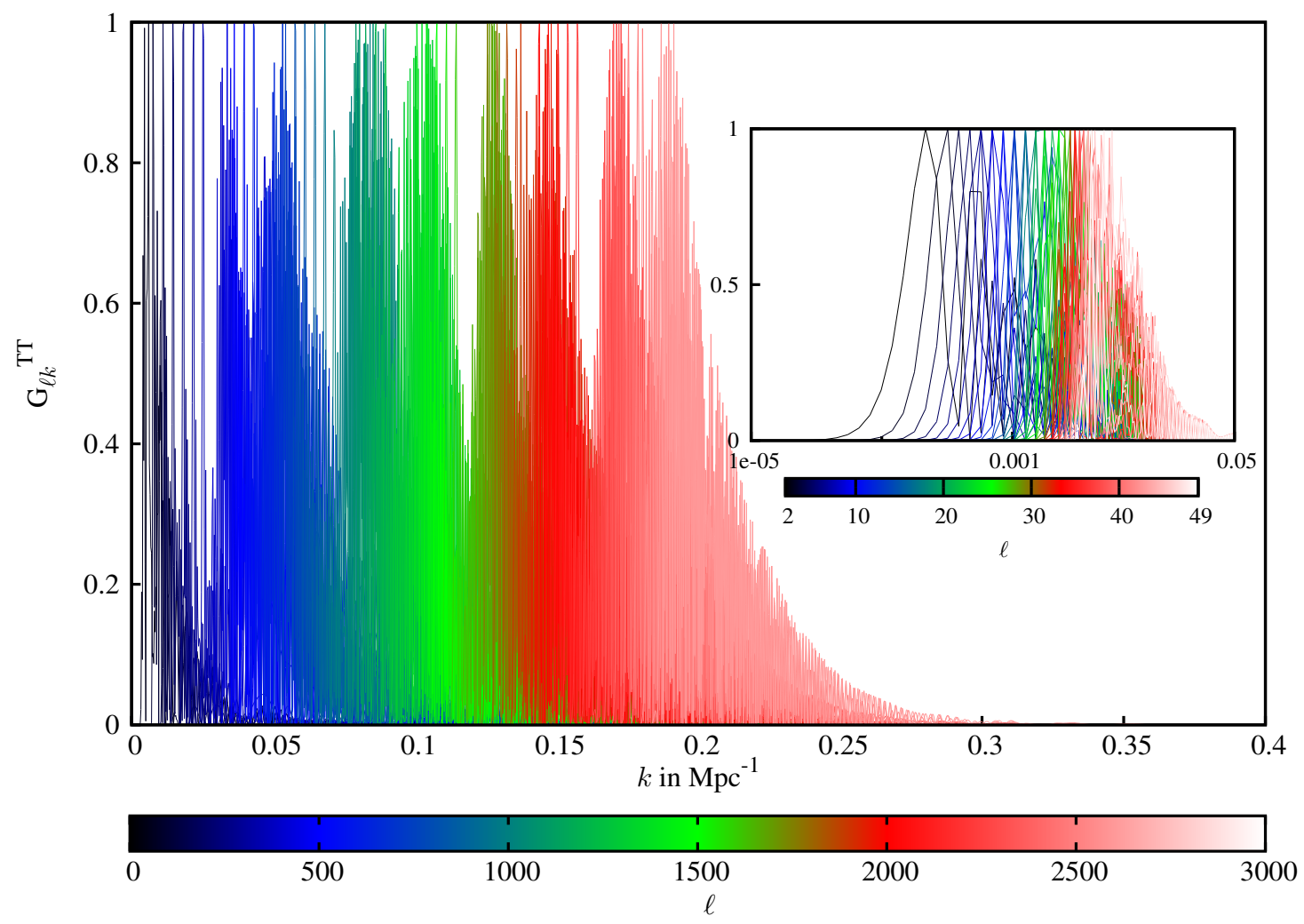

Figure 1. The radiative transport kernel (appearing in Eq. 2.1) as a function of wavenumber is plotted. The colorbar at bottom represents the multipole moment $(\ell)$ ranging from 50 to 2500 . The inset contains the plot for $\ell=2-49$ in logarithmic scale in $k$.

In order have a clean angular power spectrum let us begin with identifying and naming individual spectra and their combinations used in this paper. Table. 1 lists the multipole range covered by individual spectrum. For simplicity to address our results we follow a nomenclature as has been indicated in the table. The largest scale angular power spectrum is denoted by $\alpha$, while the two intermediate scale angular power spectrum are identified by $a(100 \mathrm{GHz})$ and $b(143 \mathrm{GHz})$. Two smallest scale angular power spectrum are marked as $1(217 \mathrm{GHz})$ and $2(143 \mathrm{GHz} \times 217 \mathrm{GHz})$. We work with 8 different combinations of the power spectrum. Starting from the largest scales the different combinations are chosen to cover the multipole range step-by-step with minimal overlap in scales. Hence the possible combinations are $\alpha, \alpha+a, \alpha+$ $b, \alpha+a+1, \alpha+a+2, \alpha+b+1, \alpha+b+2$. Finally we choose the 8'th combination to be the entire spectrum, denoted by $\alpha+a+b+1+2$. For our analysis throughout the paper we shall refer to this nomenclature.

We now present the complete algorithm of modified Richardson-Lucy (MRL), particularly designed for the analysis with Planck, though the main algorithm being similar to [3, 7]. MRL algorithm is an iterative method, where the PPS at $i+1^{\prime}$ th iteration, $P_{k}^{(i+1)}$, is given as a modification to the PPS at $i$ 'th iteration, $P_{k}^{(i)}$ as has 


\begin{tabular}{|l|l|l|l|}
\hline \hline Our symbol & Spectra & Multipoles $(\ell)$ & Scales \\
\hline$\alpha$ & low- $\ell$ & $2-49$ & Largest scales \\
\hline a & $100 \mathrm{GHz} \times 100 \mathrm{GHz}$ & $50-1200$ & Intermediate scales \\
\hline $\mathrm{b}$ & $143 \mathrm{GHz} \times 143 \mathrm{GHz}$ & $50-2000$ & Intermediate scales \\
\hline 1 & $217 \mathrm{GHz} \times 217 \mathrm{GHz}$ & $500-2500$ & Small scales \\
\hline 2 & $143 \mathrm{GHz} \times 217 \mathrm{GHz}$ & $500-2500$ & Small scales \\
\hline \hline
\end{tabular}

Table 1. Our nomenclature of referring to individual Planck spectra. We have divided the complete angular power spectra from Planck in largest, intermediate and smallest cosmological scales.

been provided in Eq. 2.2. $\widetilde{G}_{\ell k}$ is the transport kernel, normalized in each $\ell \cdot \mathcal{C}_{\ell}^{\mathrm{T}(i)}$ is the theoretical angular power spectrum corresponding to the PPS at $i$ 'th iteration, $P_{k}^{(i)}$.

$$
\begin{aligned}
P_{k}^{(i+1)}-P_{k}^{(i)} & =P_{k}^{(i)} \times \sum_{\nu}\left[\sum_{\ell=\ell_{\min }^{\nu}}^{\ell_{\max }^{\nu}(\leq 1900)} \frac{1}{g_{\nu}(\ell)} \widetilde{G}_{\ell k}\left\{\left(\frac{\mathcal{C}_{\ell}^{\mathrm{D}_{\nu}^{\prime}}-\mathcal{C}_{\ell}^{\mathrm{T}(i)}}{\mathcal{C}_{\ell}^{\mathrm{T}(i)}}\right) \tanh ^{2}\left[Q_{\ell}\left(\mathcal{C}_{\ell}^{\mathrm{D}_{\nu}^{\prime}}-\mathcal{C}_{\ell}^{\mathrm{T}(i)}\right)\right]\right\}_{\text {unbinned }}\right. \\
& \left.+\sum_{\ell=\ell_{\min }^{\nu}(>1900)}^{\ell_{\max }^{\nu}} \frac{1}{g_{\nu}^{\prime}(\ell)} \widetilde{G}_{\ell k}^{\prime}\left\{\left(\frac{\mathcal{C}_{\ell}^{\mathrm{D}_{\nu}^{\prime}}-\mathcal{C}_{\ell}^{\mathrm{T}(i)}}{\mathcal{C}_{\ell}^{\mathrm{T}(i)}}\right) \tanh ^{2}\left[\frac{\mathcal{C}_{\ell}^{\mathrm{D}_{\nu}^{\prime}}-\mathcal{C}_{\ell}^{\mathrm{T}(i)}}{\sigma_{\ell}^{\mathrm{D}_{\nu}}}\right]^{2}\right\}_{\text {binned }}\right]
\end{aligned}
$$

$\mathcal{C}_{\ell}^{\mathrm{D}_{\nu}^{\prime}}$ is the clean angular power spectrum (i.e. the data) from the spectrum $\nu$. Clean angular power spectrum refers to the spectrum obtained after calibrating and subtracting the foreground power spectrum from each of the raw angular power spectrum, $\mathcal{C}_{\ell}^{\mathrm{D}_{\nu}}$ provided by Planck. Foreground and calibration effects are calculated from CAMspec $[18,19]$ for the best fit foreground and calibration parameters obtained from Planck analysis for the baseline model. Moreover, we calculate the lensed angular power spectrum $\left(\mathcal{C}_{\ell}^{\text {Lensed }}\right)$ and un-lensed power $\operatorname{spectrum}\left(\mathcal{C}_{\ell}^{\text {un-lensed }}\right) \ddagger$ for the best fit baseline model. We define the lensing template as their difference following Eq. 2.3

$$
\mathcal{C}_{\ell}^{\text {Lens-template }}=\mathcal{C}_{\ell}^{\text {Lensed }}-\mathcal{C}_{\ell}^{\text {un-lensed }}
$$

Hence, correspond to the $\mathcal{C}_{\ell}^{\mathrm{T}}$ in Eq. 2.1 and 2.2, we use $\mathcal{C}_{\ell}^{\mathrm{D}_{\nu}^{\prime}}$ which we obtain upon subtracting the lensing template after cleaning the foregrounds from raw data. Note that throughout the paper we use the same convention apart from subsection 3.2 where we check the lensing effect with and without subtracting the lensing template.

\footnotetext{
$\ddagger$ The lensing effect is calculated using CAMB assuming curved sky correlation function method.
} 
The term $g_{\nu}(\ell)$ in Eq. 2.2 appears as a degeneracy factor and counts the number of spectra contributing to a specific multipole $\ell$. For instance, while analyzing the combination $\alpha+a+b+1+2$, for $\ell=2-49$ only the spectrum $\alpha$ contributes which makes $g_{\nu}(\ell)=1$ and at $\ell=500$ where four spectra $(a, b, 1$ and 2$)$, contribute $g_{\nu}(\ell)$ becomes 4. Note that Eq. 2.2 breaks the modification to the PPS in 2 parts, namely binned and un-binned similar to [3]. Due to noise we get negative $\mathcal{C}_{\ell}$ 's from the data where the signal-to-noise ratio is very low that is theoretically impossible. With negative angular power spectrum the MRL algorithm fails to work as it is designed to work for positive definite matrices and hence we need to work with unbinned and binned data in a combined analysis as in [3]. We find that $\mathcal{C}_{\ell}^{\mathrm{D}_{\nu}^{\prime}}$ starts picking up negative data points after $\ell=1900$ (starting from $b$ ). Hence, we fix $\ell=1900$ to be the transition point from un-binned to binned data analysis. After multipole 1900 we bin the clean data from $b, 1$ and 2 with $\ell_{\mathrm{bin}}=50$ such that the binned data points beyond that are certainly positive. Similar to the unbinned analysis $g_{\nu}^{\prime}(\ell)$ represents the degeneracy factor for the binned analysis. $\widetilde{G}_{\ell k}^{\prime}$ is the binned radiative transport kernel with same binning width $\left(\ell_{\text {bin }}=50\right)$. The tanh factors in both the parts of Eq. 2.2 represent the convergence factors introduced in $[9,10]$. The term $Q_{\ell}$ is given by Eq. 2.4,

$$
Q_{\ell}=\sum_{\ell^{\prime}}\left(C_{\ell^{\prime}}^{\mathrm{D}^{\prime}}-C_{\ell^{\prime}}^{\mathrm{T}(i)}\right) \mathrm{COV}^{-1}\left(\ell, \ell^{\prime}\right)
$$

where, $\operatorname{COV}\left(\ell, \ell^{\prime}\right)$ represents the error covariance matrix. Ideally the full covariance matrix should be used in the analysis, however, in our analysis we shall only use the diagonal terms of the covariance matrix to optimize between computational expense and insignificant improvement in results. For binned analysis after $\ell=1900$ we use the $\sigma_{\ell}^{\mathrm{D}_{\nu}}$ as the error bars in spectra $\nu$ obtained after computation of the errors for the binned data. Note that due to asymmetric errors for low- $\ell$ data in $\alpha$ the algorithm 2.2 is modified. For $i$ 'th iteration, if the $\mathcal{C}_{\ell}^{\mathrm{T}}$ is below the data, the algorithm selects the error-bar at below to work with and it chooses the upper error-bar if the opposite happens.

Before continuing to the next section we would like to note a few points. Throughout our analysis we shall only use the publicly available Planck likelihood code [19]. The high- $\ell$ likelihood is obtained through CAMspec and the low- $\ell$ likelihood is estimated by commander which are available in public domain. For calculation of angular power spectrum we have used CAMB [20, 21]. We have developed a new code for MRL with Planck data and used it as an add-on of CAMB. The number of $k$-points used for the convolution and the $k_{\min }$ and $k_{\max }$ depend on the multipoles used for the reconstruction. For example, when we use $\alpha+a+b+1+2$, we work with $\sim 2700$ wavenumbers between $k_{\min }=7 \times 10^{-6} \mathrm{Mpc}^{-1}$ and $k_{\max }=0.44 \mathrm{Mpc}^{-1}$. This is a conservative bound where the transport kernel becomes negligible to contribute in the convolution integral. After the reconstruction the likelihood is obtained upon adding the same lensing-template which has been subtracted from the data and use the same foreground and calibration parameters from which the foreground power spectrum was calculated. While comparing with WMAP data we have used the complete WMAP-9 likelihood code supplied by WMAP [22]. 


\section{Applications and results}

In this section we shall discuss the different applications of the reconstruction as has been pointed out in the introduction.

\subsection{Consistency with WMAP data}

We begin with by discussing the consistency of the Planck and WMAP-9 data. Checking this consistency has become an important issue since we find the cosmological parameters from WMAP-9 and Planck differ significantly $[4,17]$ and the Planck angular power spectrum is $\sim 2.5 \%$ lower $[18,23]$ than the WMAP in all the scales probed by WMAP. We performed a consistency check between the two data using Crossing statistic [23] and found that disallowing an amplitude shift, two data disagree with each other at worse than $3 \sigma$ confidence.

In this paper we present a Planck spectrum dependent check of consistency between Planck and WMAP-9 (For other works, see [23, 24]). Using the radiative transport kernel obtained from Planck best fit parameters we obtain a PPS using Eq. 2.2. In this section we shall use the PPS obtained after 50 iteration. Using that PPS and the same kernel we shall check how well we can fit the WMAP-9 data. Of course, through this method we can only check a relative agreement/disagreement of Planck spectra with WMAP data. Given a set of cosmological parameters MRL reconstructs a PPS that contains the features and random noise in the data. As we do not expect the noise in Planck data to match with WMAP we implement a Gaussian smoothing algorithm following [3].

$$
P_{k}^{\text {Smooth }}=\frac{\sum_{\tilde{k}=\mathrm{k}_{\min }}^{\mathrm{k}_{\max }} P_{\tilde{k}}^{\mathrm{Raw}} \times \exp \left[-\left(\frac{\log \tilde{k}-\log k}{\Delta}\right)^{2}\right]}{\sum_{\tilde{k}=\mathrm{k}_{\min }}^{\mathrm{k}_{\max }} \exp \left[-\left(\frac{\log \tilde{k}-\log k}{\Delta}\right)^{2}\right]}
$$

Using Eq. 3.1 we smooth the raw PPS $P_{\tilde{k}}^{\text {Raw }}$ and get a smooth PPS $P_{k}^{\text {Smooth }}$ depending on the smoothing width $\Delta$. For our analysis in this section we shall use a constant smoothing width at all scales of interest. As a function of smoothing width $\Delta$ we examine the WMAP-9 likelihood of the PPS obtained from different combinations of Planck spectra. To check the amplitude difference we have allowed the overall amplitude of the PPS to vary from $90 \%$ to $110 \%$. We shall define the amplitude shift by factor $A$, which ranges from 0.9 to 1.1 .

In Fig. 2 and Fig. 3 we plot our results for different combinations of Planck spectra. The plots at the left panel contain the $-\ln \mathcal{L}(\mathcal{L}$ refers to likelihood) from Planck and WMAP-9 as a function of the $\Delta$. The red and blue horizontal straight lines represent the $-\ln \mathcal{L}$ from Planck and WMAP-9 for best fit baseline model respectively. The red and the blue curves indicate the Planck and WMAP-9 likelihood from the PPS reconstructed from Planck for the corresponding combinations of spectra. The green curve represents the best likelihood to WMAP-9 from the same reconstructed PPS with allowing an overall amplitude shift. Note that apart from $\alpha$ in all the cases from $\Delta \simeq 0.01$ the PPS fits the Planck data worse than power law PPS. The reason behind 
this is the following. The MRL algorithm fits the noise in the data along with possible features. A smoothing of the PPS with higher smoothing width smears out non-local features with higher frequencies resulting in worse fit to the likelihood compared to power law spectrum. Thereby we shall give importance to the results obtained till the value of $\Delta$ we get a better fit to the Planck data compared to power law model as till that point the combination of smoothing and the MRL work well. Plots at the right panel represent the amplitude factor $A$ which provides best fit to the WMAP-9 data, as a function $\Delta$. The red shaded regions represent the band of amplitude factor where we get better fit to WMAP-9 compared to power law best fit. Absence of the red band in a combination of Planck spectra indicates that even with an overall amplitude shift the PPS from that particular combination fails to provide a better fit to WMAP-9 w.r.t. to power law best fit. The green curve represent the best fit, $A$. The blue horizontal line represents $A=1$, no amplitude shift.

Below we list the results as has been indicated in Fig. 2 and Fig. 3 at different scales.

- Largest scales $(\alpha)$ : In this particular case, the left plot provides the $-\ln \mathcal{L}$ from commander from Planck (from $\ell=2-49$ ) and low- $\ell$ likelihood from WMAP9 (from $\ell=2-32$ ). Note that the reconstructed PPS from $\alpha$ is able to fit the WMAP-9 low- $\ell$ likelihood without an overall amplitude shift. Moreover the broad red band in the right panel indicates that the reconstructed PPS from Planck can fit WMAP-9 data better than power law even with overall $10 \%$ amplitude shift. This reflects that low- $\ell$ features ranging from $\ell=2-32$ are similar in both the surveys. However, the green line, that represents the best fit, indicates approximately $4 \%$ increment in power provides the best fit to WMAP-9.

\section{- Largest + intermediate scales:}

The reconstructed PPS from $\alpha+a$ provides a better fit to WMAP-9 complete datasets without any amplitude shift. Although the best fit to WMAP-9 is obtained by increasing the power around $2.5 \%$, as indicated by the green line. This fact indicates that the features in WMAP-9 and in Planck $100 \mathrm{GHz}$ spectrum (combined with with low- $\ell$ ) agree. Moreover there is a clear mismatch in amplitude between the two and the WMAP-9 data is significantly higher in amplitude (the amplitude band does not extend below 1).

For $\alpha+b$, we notice that even allowing the amplitude shift we are unable to fit the WMAP-9 data better than power law with the reconstructed PPS obtained from the $143 \mathrm{GHz}$ (with low- $\ell$ ) spectrum. We expect to revisit this issue with taking into account the correlation between multipoles and having detailed analysis [25]. The best fit green line in the right panel here indicates amplitude difference similar to the case of $\alpha+a$.

- Largest + intermediate + smallest scales ${ }^{\S}$ :

${ }^{\S}$ Here by smallest scales we refer to the smallest scale probed by Planck i.e. till $\ell=2500$ 
The primordial spectra reconstructed from $\alpha+a+1$ and $\alpha+a+2$ show results similar to the case of $\alpha+a$. We find that the inclusion of 1 and 2 spectra does not change the results (obtained for $\alpha+a$ ) significantly. This is an expected result since 1 and 2 have less overlap in multipoles with WMAP-9 compared to $a$ and $b$. However, the improvement in fit to WMAP-9 over power law degrades marginally without an amplitude shift. As before, the best fit is obtained with $2.5 \%$ shift in amplitude.

Results from $\alpha+b+1$ and $\alpha+b+2$ is dominated by spectrum $b$ since it has the larger overlap with WMAP-9 probed scales $(\ell=2-1200)$ than 1 and 2 . We find similar results as in $\alpha+b$.

- Using the complete Planck spectra: Upon using the complete Planck spectra, we find that $\alpha+a+b+1+2$ shows behavior similar to $\alpha+a, \alpha+a+1$ and $\alpha+a+2$. The inclusion of $b$ degrades the result slightly since we find that PPS from $\alpha+a+b+1+2$ fails to fit the WMAP-9 data better than power law without an overall amplitude shift. Note that the amplitude band is now strictly greater than unity. The best fit $A$ is found to be approximately $2.4 \%-2.5 \%$.

From all the analyses above, we find $\alpha$ and $a$ (which are the most relevant spectra to be compared with WMAP) are consistent with WMAP-9 data. Combining all spectra we find WMAP-9 and Planck are in agreement allowing 2.4\%-2.5\% amplitude shift. Although the nature of investigation is different, this result is consistent with the conclusion of [23]. 

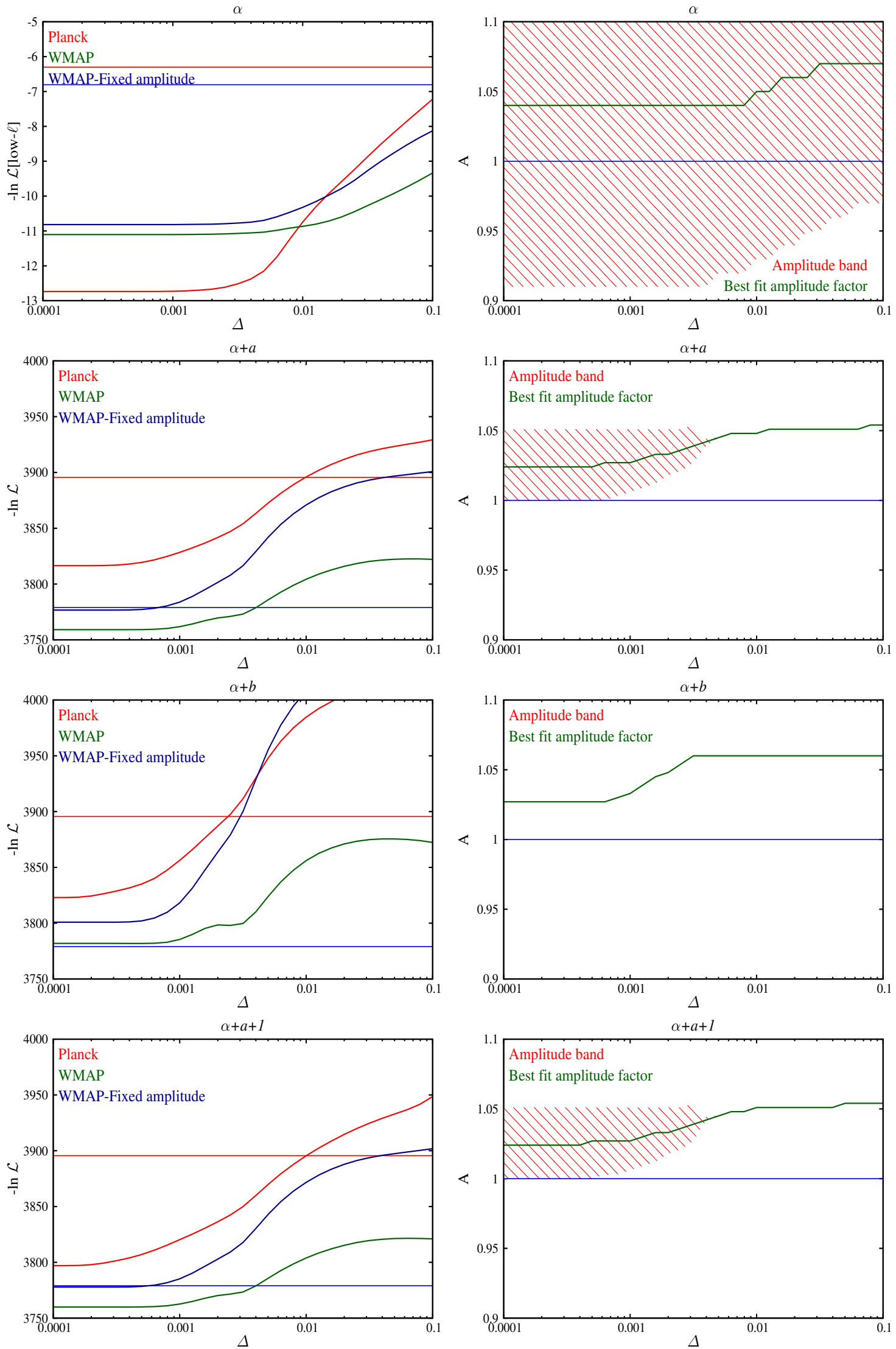

Figure 2. [Left] The $-\ln \mathcal{L}$ obtained from Planck (in red) and WMAP-9 (in green and blue) for reconstructed PPS as a function of smoothing width. Red and blue straight lines represent the power law best fit $-\ln \mathcal{L}$ from Planck and WMAP-9 respectively. Green line represents the best fit WMAP-9 $-\ln \mathcal{L}$ obtained upon comparing the reconstructed PPS from Planck, allowing an overall amplitude shift. The blue line corresponds to WMAP- $9-\ln \mathcal{L}$ obtained from the reconstructed PPS from Planck without an overall amplitude shift. [Right] The region of amplitude factor, $A$, to the PPS where we get better fit to WMAP-9 data from reconstructed PPS compared to power law PPS (red shaded area) and the value of $A$ which provides the best fit to WMAP-9 data(green line). 

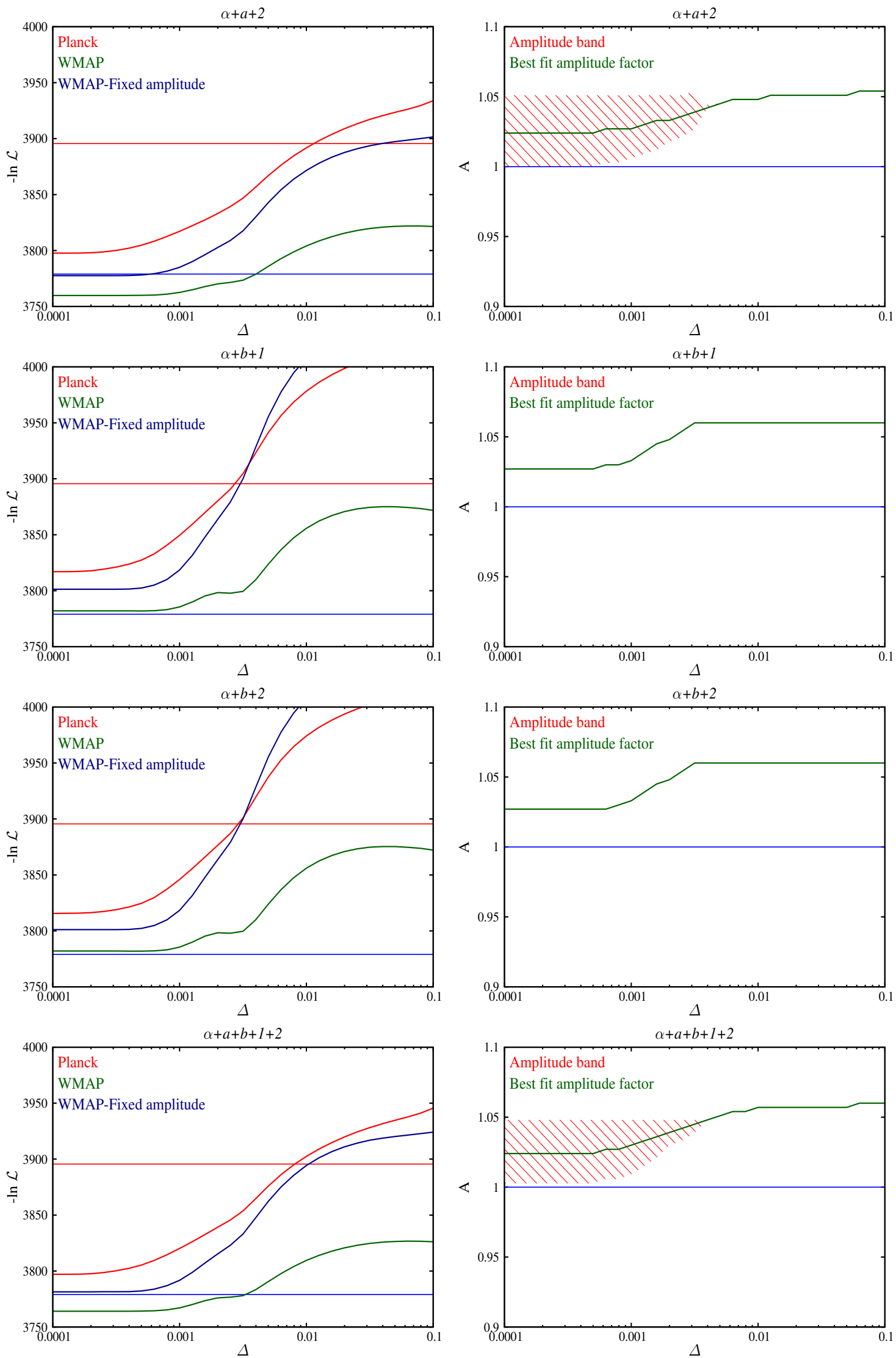

Figure 3. [Left] The $-\ln \mathcal{L}$ obtained from Planck (in red) and WMAP-9 (in green and blue) for reconstructed PPS as a function of smoothing width. Red and blue straight lines represent the power law best fit $-\ln \mathcal{L}$ from Planck and WMAP-9 respectively. Green line represents the best fit WMAP-9 $-\ln \mathcal{L}$ obtained upon comparing the reconstructed PPS from Planck, allowing an overall amplitude shift. The blue line corresponds to WMAP- $9-\ln \mathcal{L}$ obtained from the reconstructed PPS from Planck without an overall amplitude shift. [Right] The region of amplitude factor, $A$, to the PPS where we get better fit to WMAP-9 data from reconstructed PPS compared to power law PPS (red shaded area) and the value of $A$ which provides the best fit to WMAP-9 data(green line). 


\subsection{Reconstruction indicates lensing}

Gravitational lensing distorts the CMB spectrum, specifically lensing can increase/decrease the power of the CMB acoustic peaks. With the detection of small scale CMB power spectrum, Planck has confirmed effect of lensing by $25 \sigma$ [6] assuming a power law PPS. With our reconstruction we demonstrate signatures of lensing in the angular power spectra. We follow the following procedures :

1. Using the MRL we reconstruct 2 PPS. For the first PPS we consider the lensing effect, i.e. we assume the detected CMB angular power spectrum is lensed. After foreground subtraction from the raw data we also subtract the lensing template (Eq. 2.3) from each spectra and obtain $\mathcal{C}_{\ell}^{\mathrm{D}_{\nu}^{\prime}}$. Performing the reconstruction with the resulting $\mathcal{C}_{\ell}^{\mathrm{D}_{\nu}^{\prime}}$ we get the PPS as $P_{\mathrm{S}}(k)^{\text {Lens }}$. The second PPS $\left(P_{\mathrm{S}}(k)^{\text {No-Lens }}\right)$ is reconstructed using $\mathcal{C}_{\ell}^{\mathrm{D}_{\nu}^{\prime}}$ obtained after foreground subtraction but without subtracting the lensing template. Hence, for the second case we assume that the data indicates no lensing effect and argue that $\mathcal{C}_{\ell}^{\mathrm{T}}$ (Eq. 2.1) along with the foregrounds are enough to address the CMB data obtained.

2. We define the difference in the PPS : $\Delta P_{\mathrm{S}}(k) \equiv P_{\mathrm{S}}(k)^{\text {Lens }}-P_{\mathrm{S}}(k)^{\text {No-Lens }}$.

3. We obtain 2 reconstructed $\mathcal{C}_{\ell}^{\mathrm{T}}$ from both the PPS. We add the lensing template back to the first $\mathcal{C}_{\ell}^{\mathrm{T}}$ obtained only and tag it as $\mathcal{C}_{\ell}^{\text {Lens }}$. The second power spectrum is named as $\mathcal{C}_{\ell}^{\text {No-Lens }}$

4. We define the difference in the angular power spectrum as, $\Delta \mathcal{C}_{\ell}^{\mathrm{TT}} \equiv \mathcal{C}_{\ell}^{\text {Lens }}-$ $\mathcal{C}_{\ell}^{\text {No-Lens }}$.

5. We examine the $-\ln \mathcal{L}$ as a function of MRL iterations for $i \leq 500$ in different combinations of Planck spectra.

If the lensing effect is significant, we can expect the first PPS can provide a better likelihood compared to the second one. Moreover, the second PPS can be expected to contain oscillations since the MRL will enforce the lensing effect to be encoded in the PPS. Hence, we can expect periodic oscillations in $\Delta P_{\mathrm{S}}(k)$. If the lensing effect is completely captured by the oscillations in the second PPS, we expect $\Delta \mathcal{C}_{\ell}^{\mathrm{TT}}$ to be zero at all scales and both the angular power spectra provide similar likelihood to the data.

In Fig. 4 and in Fig. 5 we plot our results. The left panels contain the $-\ln \mathcal{L}$ as a function of MRL iteration $₫$ for the PPS obtained with (red) and without (blue) considering the lensing effect. Apart from the reconstruction from $\alpha$ in all other cases the $-\ln \mathcal{L}$ is plotted for the complete likelihood (using commander and CAMspec). For $\alpha$ we have plotted the results from commander only. Middle panels contain $\Delta P_{\mathrm{S}}(k)$ obtained after 100 iterations. Right panels contain $\Delta \mathcal{C}_{\ell}^{\mathrm{TT}}$ (red) obtained after 100 iterations and the reference lensing template (blue-dashed) used in the analysis.

Below we highlight the results obtained in different combinations of spectra, following Fig. 4 and 5.

\footnotetext{
INote that only for the first case $\alpha$ we calculate the likelihood from the commander. For any other spectra combinations we calculate the likelihood from both commander and CAMspec.
} 

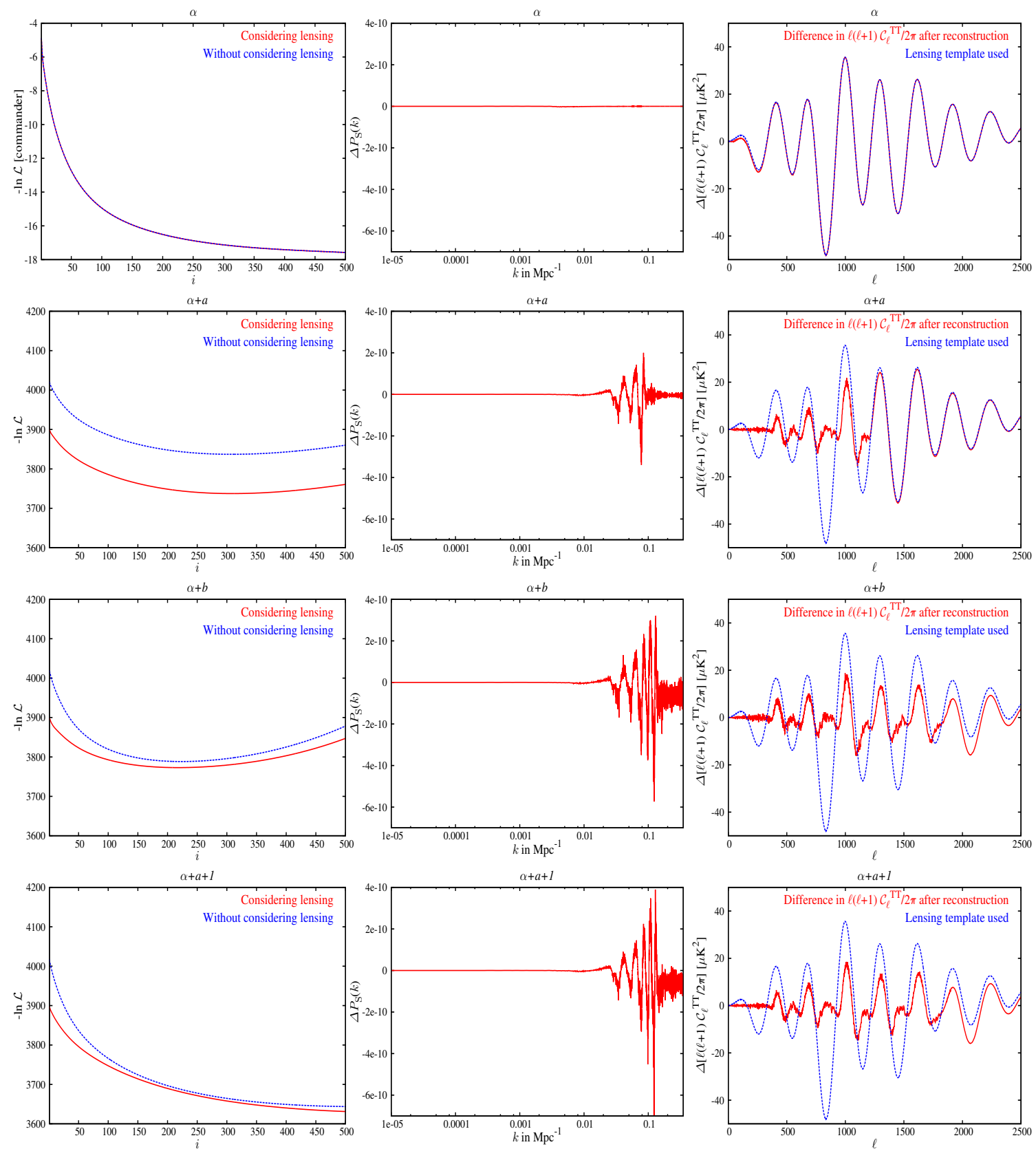

Figure 4. [Left] $-\ln \mathcal{L}$ from the reconstructed PPS with(red) and without(blue) considering the lensing effect. [Middle] The difference in PPS obtained with and without considering lensing after 100 iterations. [Right] The difference in $\mathcal{C}_{\ell}$ 's obtained from reconstruction (for 100 iterations) with and without considering lensing effect (red) and the reference lensing template used in the analysis (blue-dashed).

- $\alpha$ : Since $\alpha$ contains angular power spectrum on largest scales only, where we expect negligible lensing, the $-\ln \mathcal{L}$ is essentially equivalent (the red and the blue curves overlap). $\Delta P_{\mathrm{S}}(k)$ is zero at all scales due to the following reasons. 

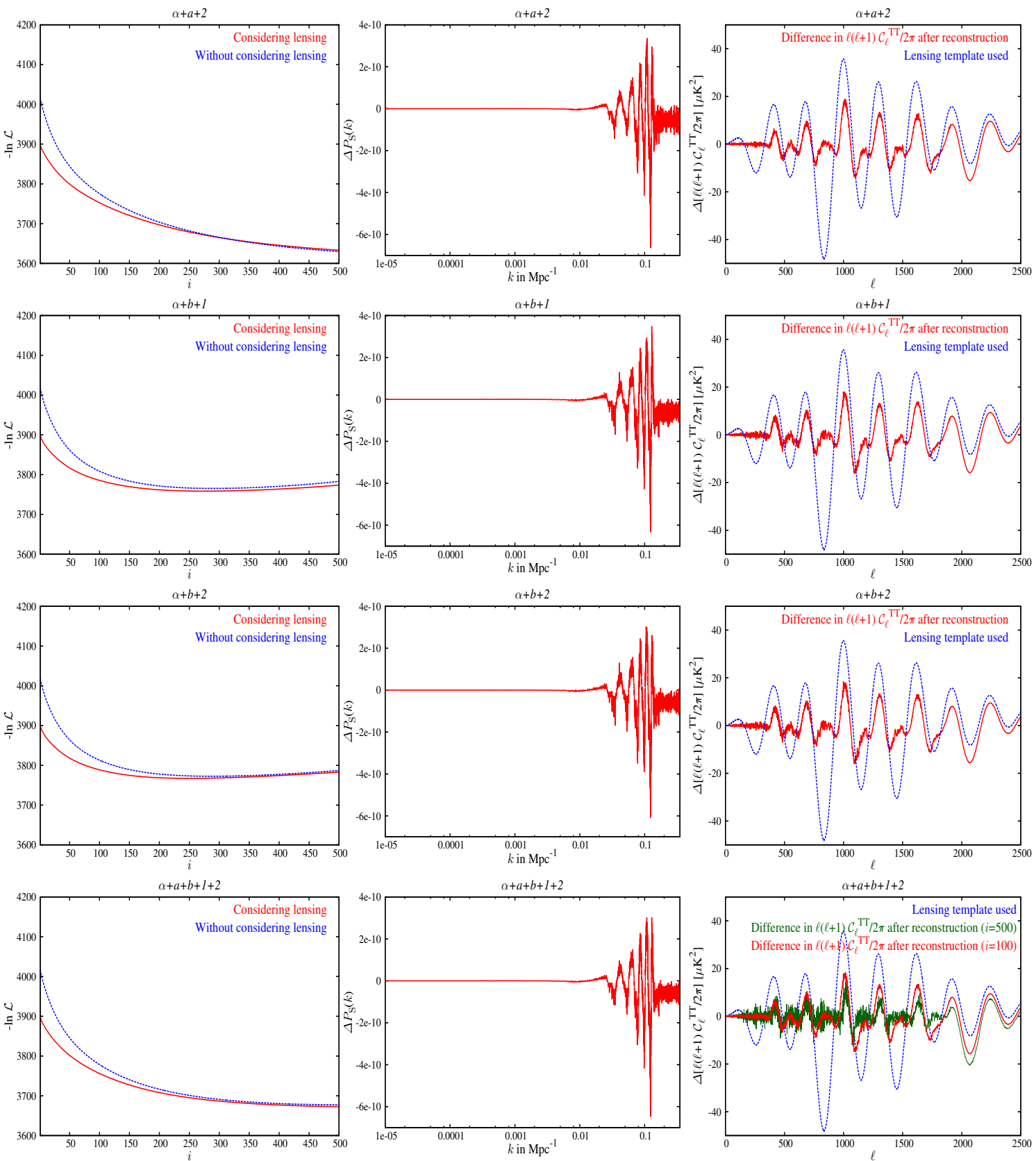

Figure 5. [Left] $-\ln \mathcal{L}$ from the reconstructed PPS with(red) and without(blue) considering the lensing effect. [Middle] The difference in PPS obtained with and without considering lensing after 100 iterations. [Right] The difference in $\mathcal{C}_{\ell}$ 's obtained from reconstruction (for 100 iterations) with and without considering lensing effect (red) and the reference lensing template used in the analysis (blue-dashed). Green curve in $\alpha+a+b+1+2$ represents same difference in $\mathcal{C}_{\ell}$ 's for 500 iterations.

At largest scales lensing is not significant and apart from large scales in both the cases the PPS is unaltered and equal to the initial choice of the PPS which we assumed to be power law. $\Delta \mathcal{C}_{\ell}^{\mathrm{TT}}$ simply follows the subtracted lensing template 
used, as expected. We get back what we have subtracted in the first case.

- $\alpha+a$ : For this combination we find a significant improvement in fit $(2 \Delta \ln \mathcal{L} \simeq$ 200) when we consider lensing effect. Hence the lensing effect becomes evident when we include data from intermediate scales. $\Delta P_{\mathrm{S}}(k)$ shows oscillations in the PPS corresponding to the acoustic peak positions in $\ell$-space. $\Delta \mathcal{C}_{\ell}^{\mathrm{TT}}$ is suppressed at scales, $\ell \leq 1200$ compared to the lensing template that highlights that a part of lensing effect is addressed by the oscillations in the PPS (prominent in $\Delta P_{\mathrm{S}}(k)$ plot). Roughly after $\ell=1200, \Delta \mathcal{C}_{\ell}^{\mathrm{TT}}$ follows the template since $a$ does not provide the data beyond that scale.

- $\alpha+b$ : Similar to $\alpha+a$, we observe lensing effect is significant in this spectra combination. However, the extent of improvement in fit has decreased compared to $\alpha+a$ since we are using 800 more data points in $b$ for the reconstruction. The use of more data increase the degeneracy between PPS features and lensing effect. $\Delta P_{\mathrm{S}}(k)$ contains even more oscillations corresponding to smaller scale lensing effects. $\Delta \mathcal{C}_{\ell}^{\mathrm{TT}}$ shows similar suppression till smaller scales. Note here the $\Delta \mathcal{C}_{\ell}^{\mathrm{TT}}$ obtained differs from the lensing template even after $\ell=2000$. This is due to the fact that the transport kernel has significant overlap in wavenumbers for $\ell=2000$ and the few hundred multipoles beyond that. Hence, when we reconstruct the PPS from $\alpha+b$ it changes the PPS at some wavenumbers which also contribute to the convolution integral for $\ell=2500$ and hence it affects the $\mathcal{C}_{\ell}$ 's beyond $\ell=2000$ to some extent.

- $\alpha+a+1, \alpha+a+2, \alpha+b+1, \alpha+b+2$ and $\alpha+a+b+1+2$ : When we add the smaller scale data we find that till $i \simeq 250$ the PPS obtained considering the lensing effect provides better likelihood. $\Delta P_{\mathrm{S}}(k)$ shows even higher oscillations in all the combinations and $\Delta \mathcal{C}_{\ell}^{\mathrm{TT}}$ 's continue to show the suppression compared to the lensing template used. Since the $\Delta \mathcal{C}_{\ell}^{\mathrm{TT}}$ is plotted for 100 'th iteration we can see that it is not completely equal to zero, since till 100 iterations lensing is providing better likelihood in all combinations that can not be mimicked by the features in the PPS. However, beyond $i \simeq 250$ the lensing effect becomes completely degenerate with PPS features. Around 500 iteration we see there is no difference in likelihood (say for $\alpha+a+b+1+2$ ). Hence we can expect no difference in $\mathcal{C}_{\ell}$ 's obtained in the two cases. We find the $\Delta \mathcal{C}_{\ell}^{\mathrm{TT}}$ obtained at $i=500$ (green curve in $\alpha+a+b+1+2$ ) is nearly zero in a broad range of multipoles before $\ell=1900$. Beyond $\ell=1900$ since we are using binned data, the flexibility of the second PPS towards mimicking lensing effect decreases.

From the results above we can certainly argue that the lensing of the CMB are reflected prominently through the reconstruction in all combinations of spectra. However, given substantially large freedom to the PPS (that can fit the noise and fluctuations significantly) it is indeed possible to mimick the lensing effect up to a very high extent (say for large iterations and complete data used in $\alpha+a+b+1+2$ ). However consideration of lensing always provide better likelihood if we restrict ourselves to low 
number of iterations where there will be less fluctuations in the reconstructed PPS. Keeping this degeneracy in mind, a proper reconstruction of the PPS must be carried out including lensing effects appropriately, otherwise the lensing can be mistakenly treated as features in the PPS.

\subsection{Features : Where are they?}

Since WMAP, the features in the PPS and their importance have been discussed widely in literature. Introduction of some particular features in the PPS can result in significant improvement in the likelihood ". However their evidence as physical effects should be addressed through proper error analysis. Hence, our first job is to hunt down these features and obtain the shape of the PPS which contain possible physical and statistical features. In [3] we have shown MRL is an excellent method to locate the features and directly provides a better likelihood to the data.

As a first step, we use MRL for different iterations $(i=1-50)$ and use different smoothing width $\Delta$, chosen randomly, (following Eq. 2.2 and 3.1) to generate a sample of PPS and $\mathcal{C}_{\ell}$ 's as plotted in Fig. 6 and in Fig. 7. In both the figures the left panels contain the samples of PPS (in different colors) obtained using the method. The colorbars at the right represent the improvement in $\chi^{2}$ from Planck likelihood code compared to best fit power law baseline model. Note that the PPS in dark blue colors are extremely smooth at small scales due to large $\Delta$ and low iterations and still provide a better fit. Right panels contain the angular power spectra corresponding to the same PPS from the left plots in same color. The data from different spectra are plotted too. The inset of the plots contain the residual angular spectra data, i.e. $\mathcal{C}_{\ell}^{\mathrm{D}_{\nu}^{\prime}}-\mathcal{C}_{\ell}^{\text {Planck best fit }}$ and residual reconstructed angular power spectra $\left(\mathcal{C}_{\ell}^{\mathrm{T}(i)}-\mathcal{C}_{\ell}^{\text {Planck best fit }}\right)^{* *}$. The dashed black lines appearing in the plots at the right correspond to the Planck best fit baseline power spectrum.

Below we highlight the features in multipole and wavenumber space obtained from different combinations of Planck spectra.

- $\alpha$ : For reconstruction obtained only from $\alpha$, we plot the colorbars that represent $-2 \Delta \ln \mathcal{L}=\Delta \chi^{2}$ only from commander. Note that the features shown in the plots can improve the likelihood by 18 compared to power law PPS. Due to quadrupole suppression $(\ell=2$ ) at largest scales ( cut-off in power compared to the best fit baseline model. The dip in power around $\ell \sim 15-30$ (specifically, near $\ell \simeq 22$ ) imprints a broad dip in the PPS around $k \simeq 0.002 \mathrm{Mpc}^{-1}$. Around $\ell \simeq 40$ we see the data indicate slight excess in power compared to the black dashed spectrum obtained from power law PPS. The power enhancement in the PPS around $k \simeq 0.0035 \mathrm{Mpc}^{-1}$ signifies this excess. However, the smooth dark blue line only picks up the cut-off around $k \simeq 2 \times 10^{-4} \mathrm{Mpc}^{-1}$

"The stand of concordance model in the light of the Planck data has been discussed in [26] where we indicated a particular damping in the small scale CMB angular power spectrum is significantly favored by Planck data

** Note that $\mathcal{C}_{\ell}^{\mathrm{T}(i)}$ 's here correspond to the reconstructed angular power spectra obtained after smoothing and adding the lensing template 
and the dip near $k \simeq 0.002 \mathrm{Mpc}^{-1}$ which indicate that compared to other features in low- $\ell$ these two features are more significant. More generally, one can say that the low- $\ell$ data indicate an overall suppression in PPS (similar to what we have found in [8]).

- $\alpha+a$ : Note that with more data from $\alpha+a$ we have started to fit the Planck angular power spectrum better $(-2 \Delta \ln \mathcal{L} \simeq 250)$ than the best fit power law PPS. The residual $\mathcal{C}_{\ell}$ 's and residual data points (in yellow) help to locate the possible features. We observe oscillations in the angular power spectrum with a dip around $\ell \sim 250-300$ in the power which correspond to a dip in PPS near $k \simeq 0.02 \mathrm{Mpc}^{-1}$. Followed by this we find a dip and a bump around $\ell \simeq$ $750-850$ which correspond to similar dip and bump in the PPS near $k \sim$ $(0.055-0.065) \mathrm{Mpc}^{-1}$.

- $\alpha+b$ : Use of the spectrum from $b$ allows us to locate the features even smaller scales. Similar to the last case, we find $\sim 200$ improvement in fit from the reconstructed PPS. The features near $\ell \sim 250-300$ and $\ell \sim 750-850$ are confirmed by $b$ spectrum too. Moreover near $\ell \sim 1800-2000$ we find a dip in the reconstructed angular power spectra ${ }^{\dagger \dagger}$. The residual data points are below zero around that region. Corresponding to that dip we find similar dip in PPS near $k \sim(0.12-0.14) \mathrm{Mpc}^{-1}$.

- $\alpha+a+1$ : The dip in the angular power spectrum around $\ell \sim 750-850$ is visible in spectrum 1 too. Near $\ell \sim 1800-2000$ we observe the feature again. Note that the residual data points from 1 indicate a more prominent dip compared to residual data from spectrum $b$ due to smaller uncertainties. This particular feature was indicated in Planck analysis [5].

- $\alpha+a+2$ : Feature around $\ell \sim 750-850$ is still visible in spectrum 2. However, we find $\ell \sim 1800-2000$ feature is not prominent in this spectrum compared to spectra $b$ and 1 (obtained from the reconstruction using angular power spectra data $\alpha+b$ and $\alpha+a+1)$.

- $\alpha+b+1$ and $\alpha+b+2$ : In these two spectra combinations we find both the features around $\ell \sim 750-850$ and $\ell \sim 1800-2000$.

- $\alpha+a+b+1+2$ : Results from reconstructions using the complete Planck spectrum are plotted in the last panels in Fig. 7. Combining all spectra, we find low- $\ell$ suppressions near $\ell=2\left(k \simeq 2 \times 10^{-4} \mathrm{Mpc}^{-1}\right)$, and dip and bump at $\ell \simeq 22$ $\left(k \simeq 0.002 \mathrm{Mpc}^{-1}\right)$ and $\ell \simeq 40\left(k \simeq 0.0035 \mathrm{Mpc}^{-1}\right)$ respectively. Apart from low- $\ell$ we also find features in the angular power spectrum near $\ell \sim 300,750-$ $850,1800-2000$.

\footnotetext{
${ }^{\dagger \dagger}$ We mention again that this particular feature is related to a systematic effect discussed in the revised version of Planck papers [5]
} 

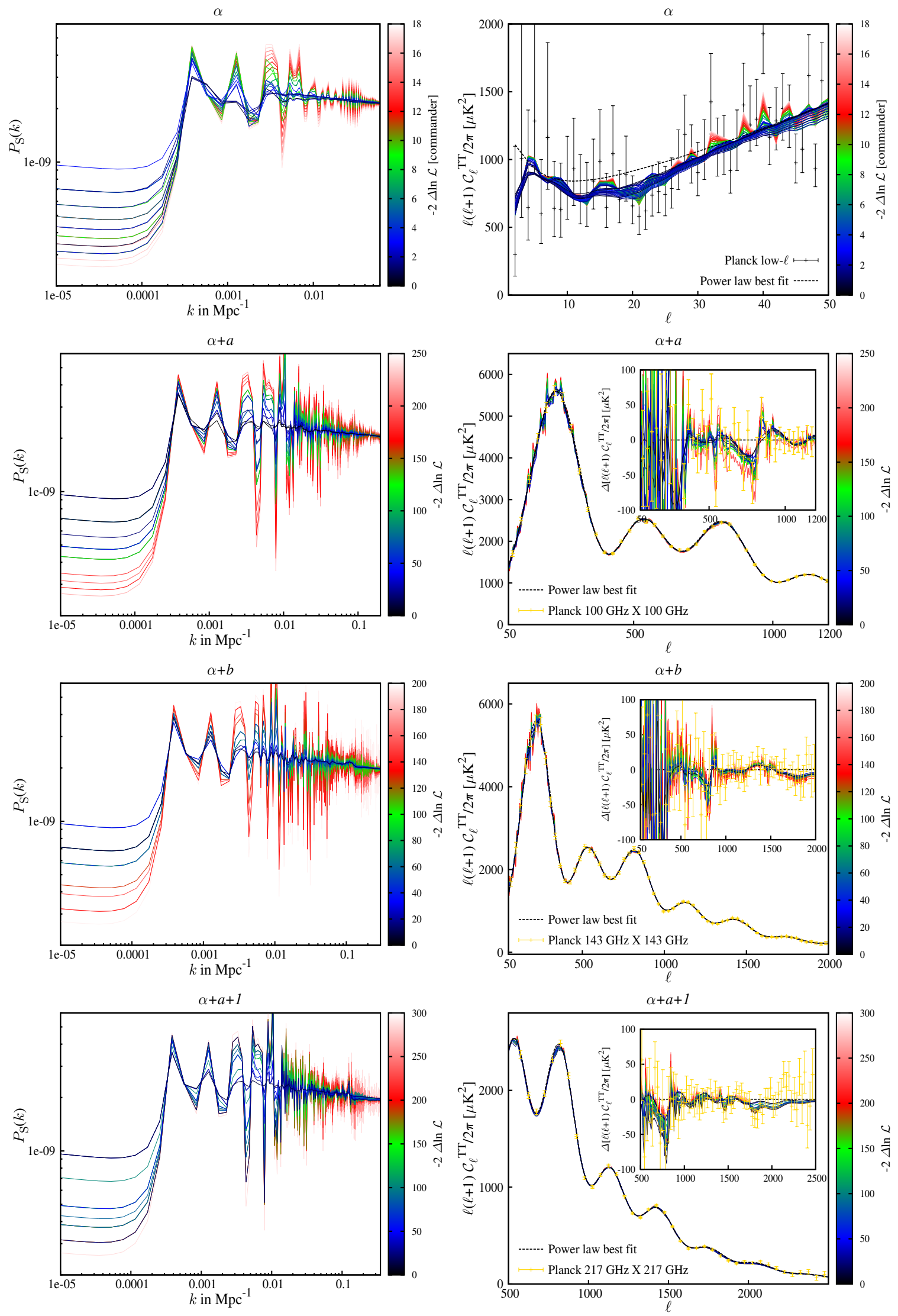

Figure 6. [Left] PPS obtained for different iterations and different smoothing and the improvement to the Planck likelihood (in $\Delta \chi^{2}=-2 \Delta \ln \mathcal{L}$ )(indicated through colorbar). [Right] Angular power spectra corresponding to the same color PPS appearing in the left plots. [Inset] Residual data with error-bars and the residual reconstructed $\mathcal{C}_{\ell}$ 's with reference to best fit Planck baseline $\mathcal{C}_{\ell}$. 

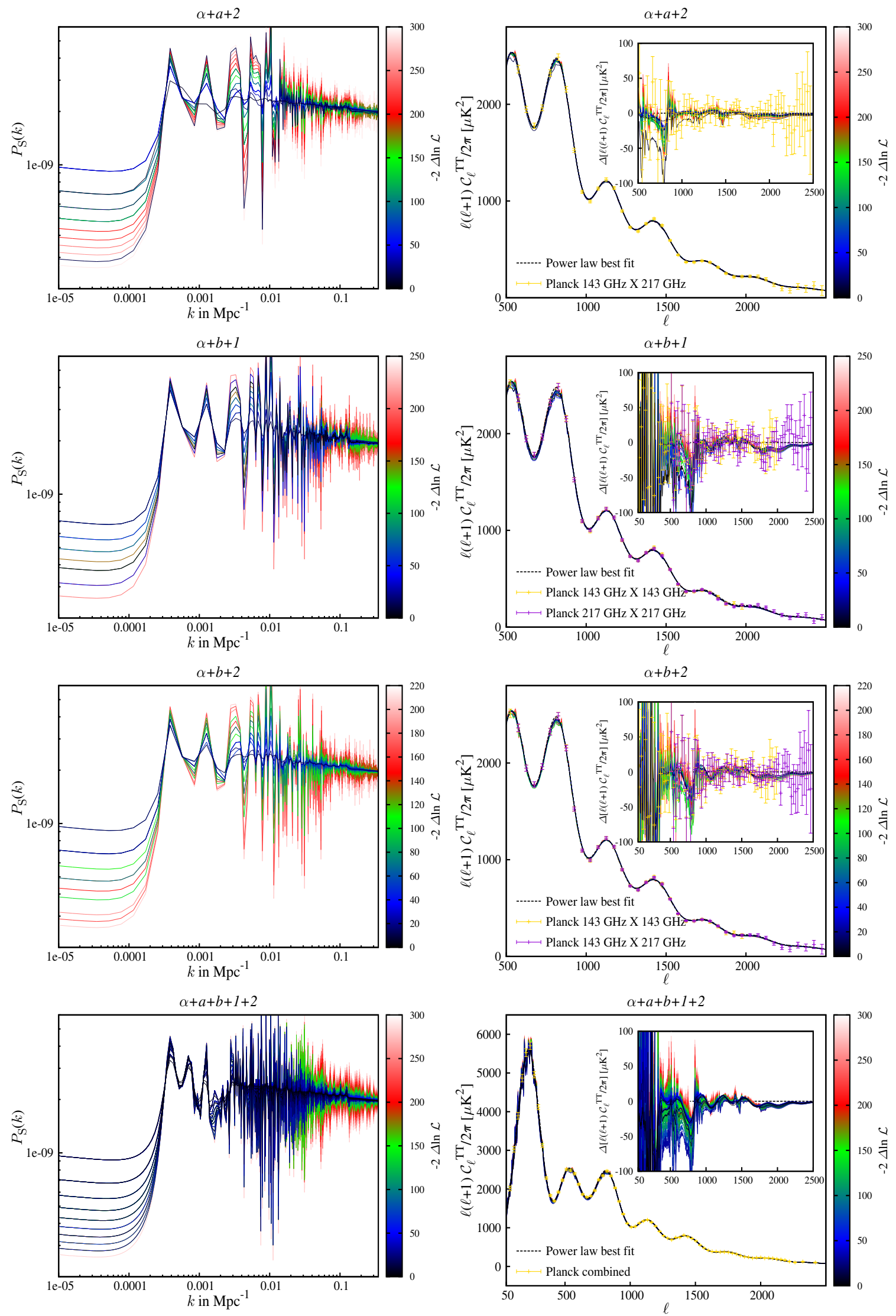

Figure 7. [Left] PPS obtained for different iterations and different smoothing and the improvement to the Planck likelihood (in $\Delta \chi^{2}=-2 \Delta \ln \mathcal{L}$ )(indicated through colorbar). [Right] Angular power spectra corresponding to the same color PPS appearing in the left plots. [Inset] Residual data with error-bars and the residual reconstructed $\mathcal{C}_{\ell}$ 's with reference to best fit Planck baseline $\mathcal{C}_{\ell}$. 


\subsection{Error estimation : Hunting down the probable physical features}

It is always expected that a standard model theory can not address all the data points and we can always expect features. However, finding a feature does not necessitates the venture to building models to explain the feature since the feature can simply be statistical rather than being a physical artifact. Moreover it can be easily understood from the reconstructed PPS in the previous section that we are getting huge improvement in fit by fitting the noise in the data. To hunt down the possible physical features we need a proper error estimation. In our analysis with WMAP-9 data [3] we had found the standard power law model is completely consistent with the data at all cosmological scales within the constraints on the PPS. With Planck data we get tighter constraints on the PPS compared to WMAP since the precision of Planck data is significantly higher than WMAP-9. Using similar formalism as in [3] we address the significance of the features and check the consistency of power law PPS with the data.

For the 8 different combinations of spectra we generate 1000 realizations of the data considering Gaussian random fluctuations. The variance of the fluctuations are equated to the diagonal term of the error covariance matrix. Since we have asymmetric errors at low- $\ell$, for $\alpha$ we use the upper-error when the random fluctuations are above the mean value and we use lower-error for the opposite. For 1000 data in each combination MRL generates 1000 PPS. In each $k$ from the reconstructed PPS we extract the most densely populated $68.3 \%$ and $95.5 \%$ region which will act as $1 \sigma$ and $2 \sigma$ constraints on the PPS. It should be kept in mind that since we are considering only one set of foreground parameters, small scale errors on the PPS are underestimated in our analysis. We expect increase in errors with the addition of foreground parameter marginalization, which is beyond the scope of this paper. To compare with the PPS reconstructions discussed in Planck papers, see [5].

In Fig. 8 we plot the $1 \sigma$ (blue) and $2 \sigma$ (cyan) errors on the PPS for different combinations of Planck spectra as a function of cosmological scales. We have used $50 \mathrm{MRL}$ iterations. In red we plot the best fit power law PPS obtained from Planck baseline model. From the figure we can directly spot the features at $k \simeq 0.002 \mathrm{Mpc}^{-1}$ $(\ell \simeq 22)$ and around $k \sim(0.12-0.14) \mathrm{Mpc}^{-1}(\ell \sim 1800-2000)$ since for both the cases power law stands outside $1 \sigma$ bounds. Note that in $\alpha+a+1$ and $\alpha+b+1$ the $k \sim(0.12-0.14) \mathrm{Mpc}^{-1}$ feature is prominent, however for $\alpha+a+2, \alpha+b, \alpha+b+2$ we find power law is within $1 \sigma$ errorband which point towards the high significance of this feature only in spectra $1(217 \mathrm{GHz} \times 217 \mathrm{GHz})$. Combining all the spectra (for $\alpha+a+b+1+2)$ we find features around $k \simeq 0.002 \mathrm{Mpc}^{-1}$ and $k \sim(0.12-0.14) \mathrm{Mpc}^{-1}$ remain prominent where the constraints on the power spectrum pushes away the power law best fit spectrum by more than $1 \sigma$. Though not visible clearly in the plots we found noticeable departures from power law PPS in few other scales for which we extend our error analysis further. 

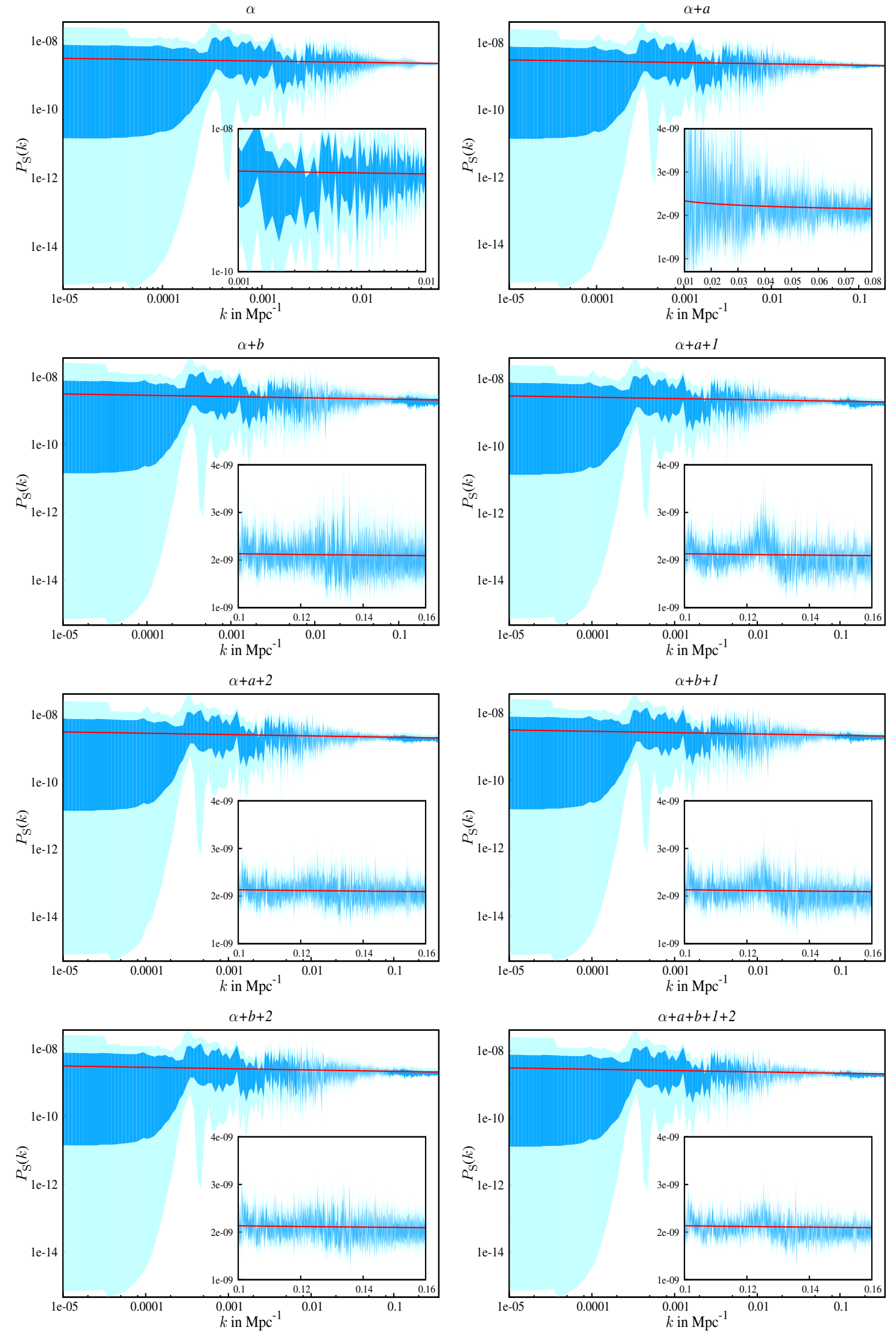

Figure 8. The $1 \sigma$ (blue) and $2 \sigma$ (cyan) error on the PPS for different combinations of Planck spectra and the best fit power law PPS (in red). 


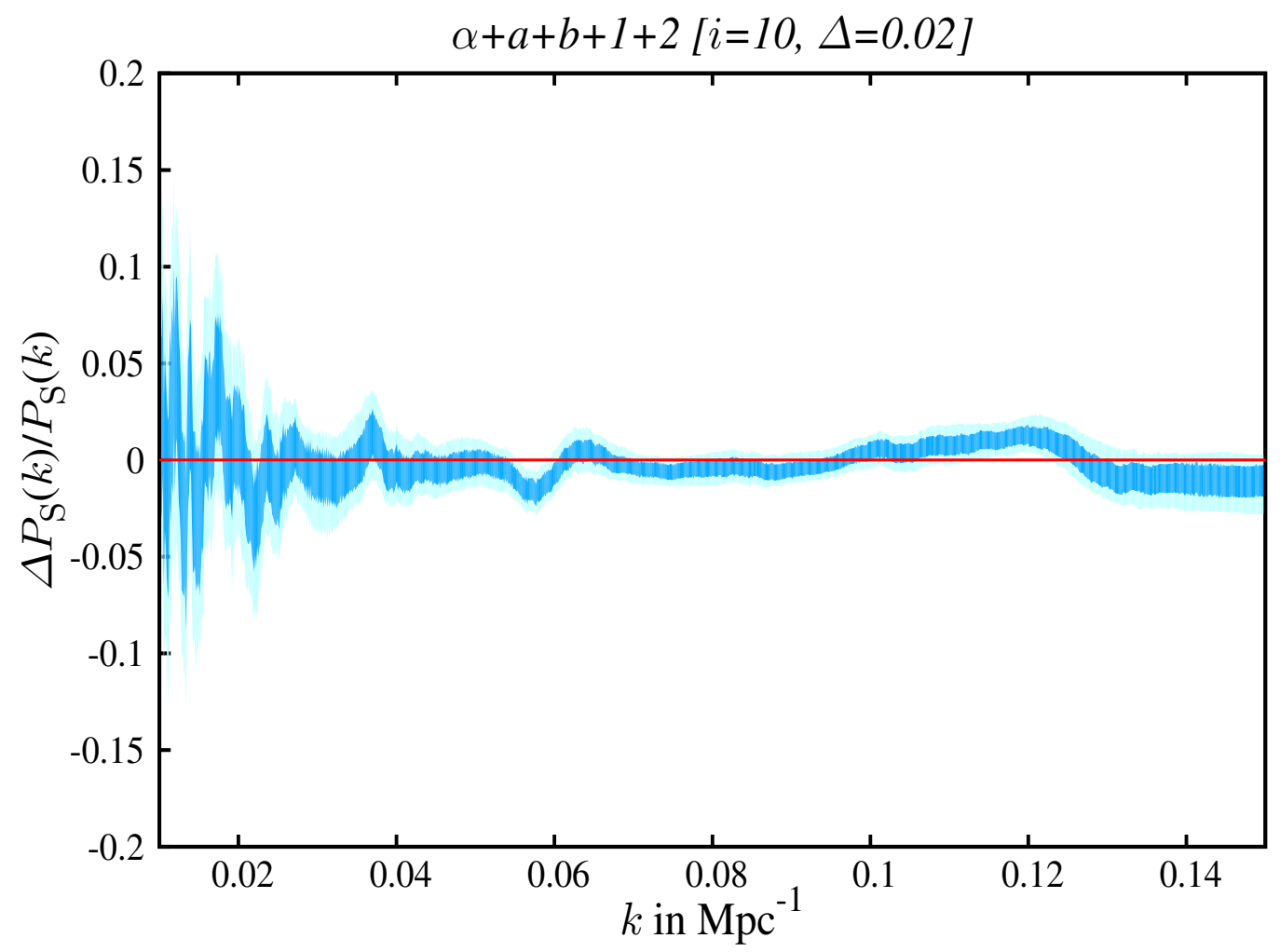

Figure 9. Errors on fractional deviations from the best fit PPS obtained using a particular combination of iterations and smoothing width, provided in the title of the plot.

Apart from the 2 features discussed above, samples in Fig. 6 and Fig. 7 also highlight oscillations near $\ell \simeq 250-300$ and a broad dip near $\ell \simeq 750-850$. These are features correspond to oscillations near $k \simeq 0.02 \mathrm{Mpc}^{-1}$ and $k \sim 0.055-0.065 \mathrm{Mpc}^{-1}$ in the PPS respectively. However, since MRL works with the un-binned and correlated data, the errors obtained are correlated too. To reduce the correlation and to highlight broad and dominant features in the data we repeat our error analysis with different iterations $(i)$ and different smoothing width $(\Delta)$, for $\alpha+a+b+1+2$. Out of few analysis we only present here one result that can show dominant features in the data within $k \sim(0.01-0.15) \mathrm{Mpc}^{-1}$ in Fig. 9. The plot in the figure represents the fractional deviations $\left(\left(\left.P_{\mathrm{S}}(k)\right|_{\text {Reconstructed }}-\left.P_{\mathrm{S}}(k)\right|_{\text {Power law best fit }}\right) /\left.P_{\mathrm{S}}(k)\right|_{\text {Power law best fit }}\right)$ from best fit power law model (for $\alpha+a+b+1+2$ ) and the significance (blue and cyan bands correspond to 1 and $2 \sigma$ errors respectively) of these deviations for $i=10$ and $\Delta=0.02$. This plot indicates $k \sim 0.055-0.065 \mathrm{Mpc}^{-1}(\ell \sim 750-850)$ and $k \sim 0.12-0.14 \mathrm{Mpc}^{-1}$ features can be significant.

We should note that the estimation of the significance of the individual reconstructed features in the PPS can be complicated due to correlations in the PPS. One can use approaches such as discrete wavelet decomposition [27] to separate features from each other and estimate the significance and importance of the reconstructed individual features. This is beyond the scope of this work and requires further inves- 
tigation [25].

The small scale features (one near $\ell \sim 1800$ ), appears due to imperfectly subtracted electromagnetic interference and has been mentioned in revised versions of Planck 2013 papers [5, 17, 18]. Having said that, we should mention that we observe the dip near $\ell \sim 1800-2000$ in spectra $b$ and 1 , however it is only spectra 1 $(217 \mathrm{GHz} \times 217 \mathrm{GHz})$ where we find the effect more than $1 \sigma$ significant. It is interesting to notice that spectrum $2(143 \mathrm{GHz} \times 217 \mathrm{GHz})$ does not indicate the feature. In this context, compare the reconstructed angular power spectra residuals and residual data points in the plot of $\alpha+a+2$ in Fig. 7. We can see that residual data points of $143 \mathrm{GHz} \times 217 \mathrm{GHz}$ in Fig. 16 of [5] also indicate similar behavior.

While we find the power law primordial power spectrum to be consistent within $2 \sigma$ at almost all cosmological scales, we also report certain localized feature around $\ell \sim 750-850$ which has the highest significance. Since compared to WMAP-9 [3], with Planck the features are found to be more significant, our analysis can encourage models of inflation with features. For example, the large scale cut-off and dip are usually addressed through a punctuation in inflation (with an inflection point in the inflaton potential) and a step in the inflationary potential. The oscillations on the other hand are very well explained by axion monodomy model. For detailed discussion on these models, see [2].

\subsection{Filtering out the noise: towards a smooth primordial power spectrum}

Towards the end of the feature hunt, it is important that we present a shape of PPS with only significant features included providing a significant improvement in fit to the Planck data over power law. While reconstructing an inflationary model from a PPS with too many oscillations is difficult and certainly unrealistic, a smooth PPS can provide the necessary information towards modification of a slow-roll inflationary potential to achieve similar PPS. In Fig. 10 we plot 2 smooth PPS obtained using the smoothing following Eq. 3.1. Using a constant smoothing width $\Delta$ we optimize the iterations and the smoothing width in order to get a significantly better likelihood than power law PPS with minimal variations in the PPS. Such a PPS is given in blue dashed line which provides 16 improvement in $\chi^{2}$ compared to the power law PPS. Moreover, the obtained errorband in our analysis provided in last subsection opens up a possibility of having error weighted PPS. The errors in the PPS capture the signalto-noise ratio in the data. Hence a smooth PPS weighted with the signal-to-noise ratio of the corresponding data contain important features in the data. In order to achieve that we smooth the PPS assuming $\Delta$ to be a proportional to the $1 \sigma$ error $\left(\ln P_{\mathrm{S}}(k) \mid 1 \sigma\right.$ up $-\ln P_{\mathrm{S}}(k) \mid 1 \sigma$ low $) \ddagger$ obtained for $\alpha+a+b+1+2$. The smooth PPS is plotted as green dashed line in Fig. 10. The green line provides 12 better fit over power law PPS. The best fit power law PPS is plotted black dashed line.

Comparing the 2 PPS with the power law model we can understand that the error weighted PPS has much less oscillations at large scales compared to the uniformly smoothed PPS and can be described by simple forms. This PPS can come in handy to

\footnotetext{
${ }^{\ddagger \ddagger} \ln P_{\mathrm{S}}(k) \mid 1 \sigma$ up/low represent the logarithmic value of the upper and lower $1 \sigma$ error on $P_{\mathrm{S}}(k)$.
} 


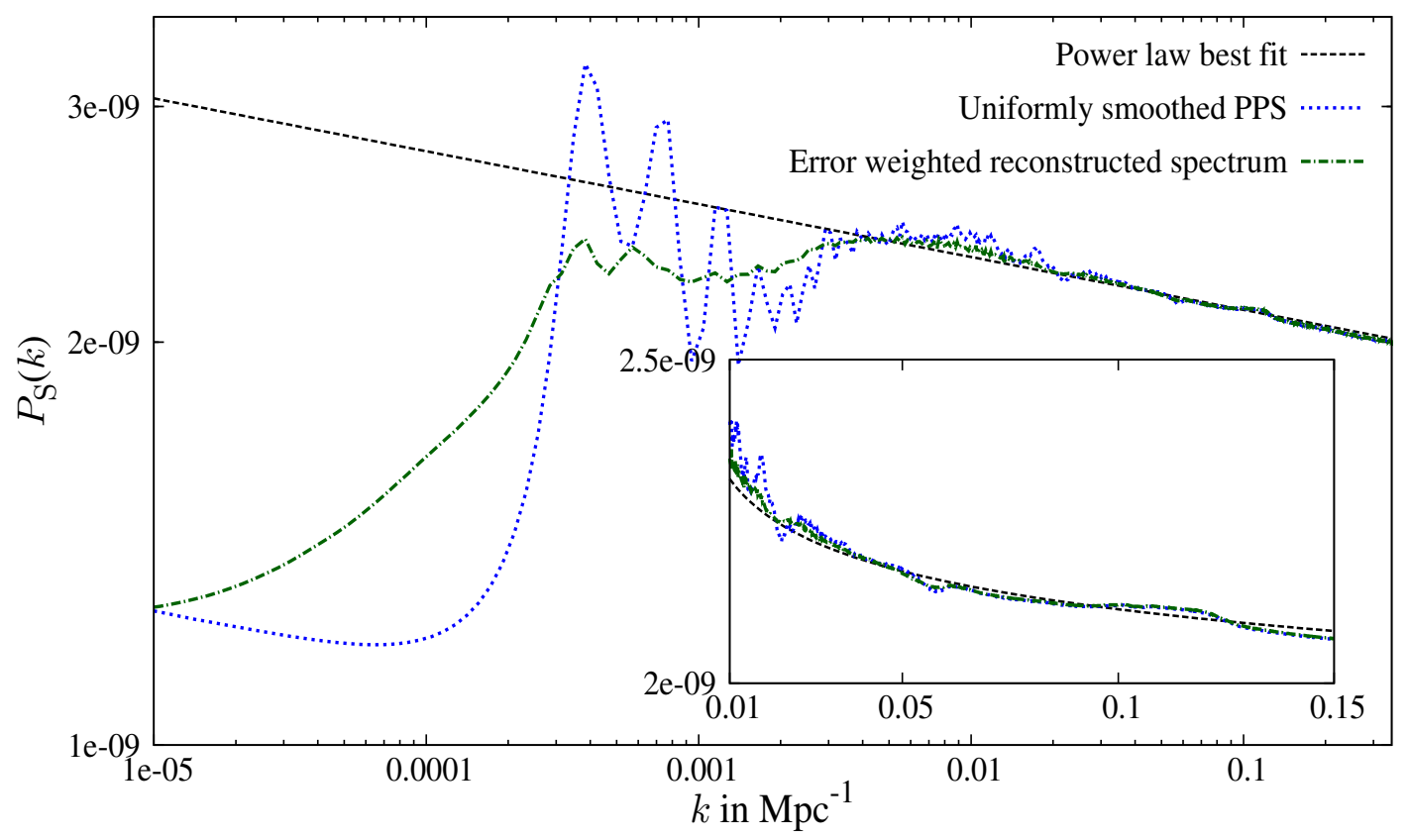

Figure 10. Smooth primordial power spectra obtained using uniform smoothing (blue dashed) and error-weighted smoothing (green dashed) in logarithmic scale. The best fit power law in dashed black is plotted as a reference. The blue and the green PPS fit the Planck angular power spectrum data better compared to power law PPS with improvement in $\Delta \chi^{2}$ of 16 and 12 respectively. The inset highlight small scale feature in linear scales.

be attempted from an inflationary potential with few parameters. Note that the green PPS indicates a blue tilt up-to $k \simeq 0.01 \mathrm{Mpc}^{-1}$ reflecting that till this scale the data does not constrain the PPS to have a necessary red tilt. This fact re-establishes our result obtained in [8] for a PPS broken in 2 bins. Oscillations around $0.02 \mathrm{Mpc}^{-1}$ is visible in blue curve. Both the green and blue PPS agrees on small scales and indicate a broad dip at $k \sim 0.055-0.065 \mathrm{Mpc}^{-1}$ (corresponding to $\ell \sim 750-850$ ) and a broad oscillation at $k \sim 0.12-0.14 \mathrm{Mpc}^{-1}$ (corresponding to $\ell \simeq 1800-2000$ ). However, due to probable systematics in the data mentioned in Planck paper in [5] immediate priority should not be given to the feature at $\ell \simeq 1800-2000$.

For the same reconstructed PPS and the power law PPS appearing in Fig. 10 we plot the corresponding $\mathcal{C}_{\ell}$ 's in plot 11 . The data points with errors are plotted in red. Power spectra and the data from low- $\ell$ are plotted in logarithmic scale and from high- $\ell$ are plotted in linear scale. Note that in apart from the large scale cut-off, all 3 spectra look identical. To identify the difference between the spectra we plot the spectra in the space residual to the Planck best fit baseline angular power spectra, i.e. we plot $\ell(\ell+1)\left(\left.\mathcal{C}_{\ell}^{\mathrm{TT}}\right|_{\text {Data/reconstructed spectrum }}-\left.\mathcal{C}_{\ell}^{\mathrm{TT}}\right|_{\text {Planck best fit }}\right) / 2 \pi$. Note that apart from the large scale cut-off, the dip near $\ell \simeq 22$ is addressed in both the spectra. Both the green and blue spectra attempts to fit the feature in the data near $\ell \sim 250-300$. Note that while the blue curve fits data with oscillations present around multipole 250-300 (with 


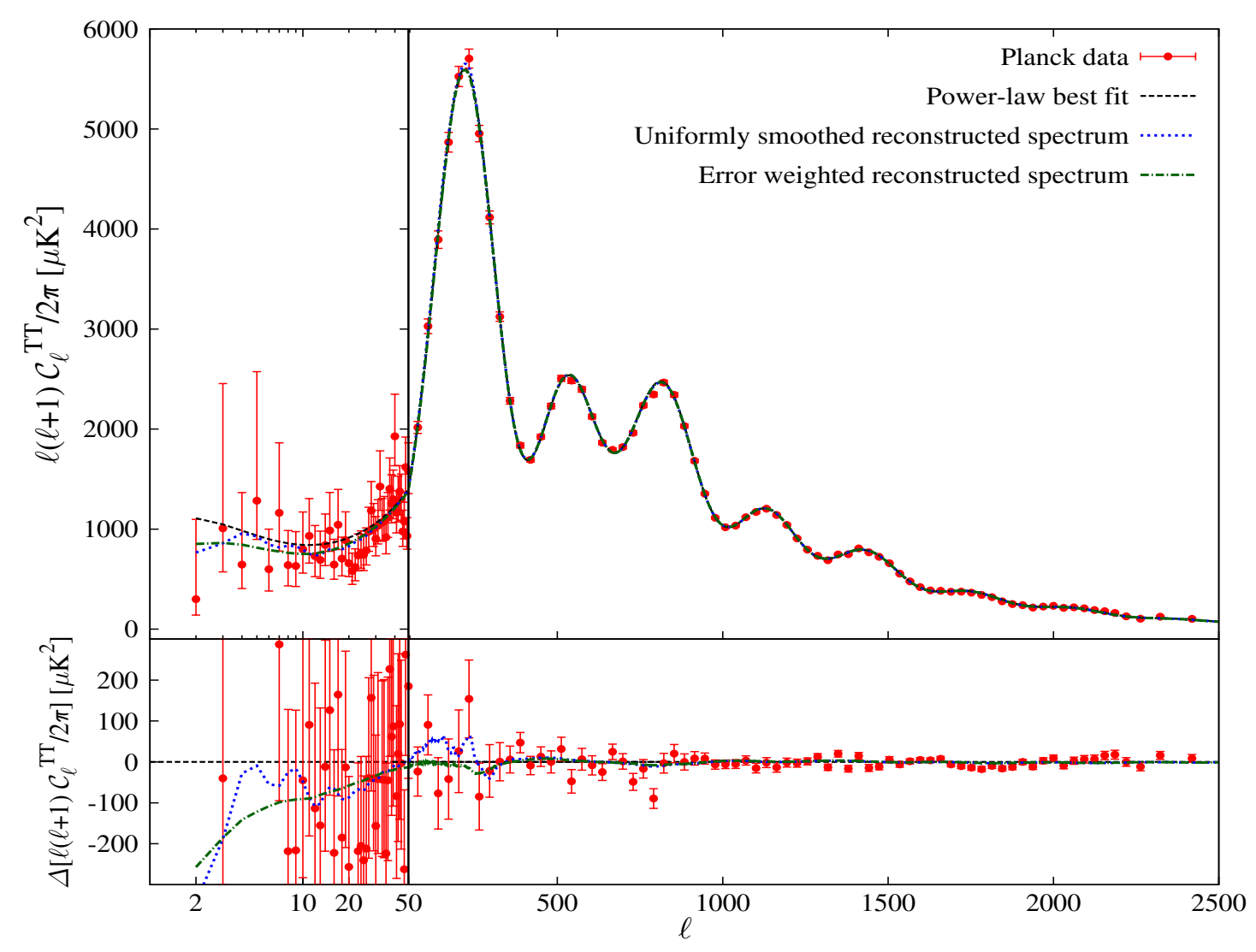

Figure 11. Binned Planck data (red) (combining all the spectrum) and the angular power spectrum obtained from power law PPS (dashed black line), uniformly smoothed reconstructed PPS (blue dashed) and error-weighted PPS (green dashed) as plotted in Fig. 10. Angular power spectrum and data from low- $\ell$ (2-49) are plotted in logarithmic scales and high- $\ell$ (50-2500) are plotted in linear scale. Residual data and the angular power spectra (with reference model as Planck best fit baseline model) for the same models are piloted at the bottom.

a pronounced dip near 300), the error weighted green spectrum gives importance to the particular dip near 300. Fig. 10 also indicates the attempts to fit features around $\ell \sim 750-850$ and $\ell \sim 1800-2000$ which are not prominently visible in this figure due to large range covered by the plot.

\section{Discussion}

In this paper we discuss about reconstruction of the primordial power spectrum (PPS) from Planck CMB data using Modified Richardson-Lucy (MRL) algorithm. Planck best fit baseline model has been used as the background cosmology and we used 8 different combinations of Planck spectra for the purpose of reconstruction. In our analysis we attempt to fit WMAP-9 data using the reconstructed PPS from Planck spectra and address the consistency of the WMAP-9 and Planck data. Our results indicate that Planck low- $\ell$ and the $100 \mathrm{GHz}$ spectra are consistent to WMAP-9 data 
allowing an overall amplitude shift. Combining all Planck spectra we show that the reconstructed PPS is consistent to WMAP-9 allowing an overall $\simeq 2.5 \%$ amplitude shift, which is in agreement with the other studies along this line [18, 23].

We do also discuss the reconstruction of the PPS studying the effect of lensing and report definitive evidence of lensing in the data for all combinations of spectra except for the case of low- $\ell$ spectrum (as expected). Our results indicate that allowing large amount of fluctuations in the form of PPS it is possible to mimic the lensing effect through features in the PPS. This degeneracy is an important issue to consider when we are looking for the features in the form of the PPS.

Using MRL algorithm we have been able to locate possible features in the form of the PPS by analyzing different combinations of the Planck spectra. We have performed an extensive error analysis using 1000 realizations of the data in all 8 combinations of the spectra to obtain realistic constraints on the form of the PPS and estimate the statistical significance of the reconstructed features. $\ell \simeq 22\left(k \simeq 0.002 \mathrm{Mpc}^{-1}\right), 300$ $\left(k \simeq 0.02 \mathrm{Mpc}^{-1}\right)$ and $750-850\left(k \sim 0.055-0.065 \mathrm{Mpc}^{-1}\right)$ features are found to be the most prominent ones, of them a dip around $\ell \sim 750-850$ with highest significance. We report that feature at $\ell \sim 1800-2000$ is evident with high significance only in 217 $\mathrm{GHz}$ spectra and apparently appears due to small systematics as has been reported by Planck [5]. In a conservative error estimation we find standard power law PPS remains consistent within $2 \sigma$ constraints in almost all scales. At the end we present two smooth form of the PPS (that can fit the data significantly better than the power law model) that can be described by relatively simple functional forms with limited number of parameters. This can be helpful for inflationary model building to address the data using alternative scenarios.

\section{Acknowledgments}

The authors would like to thank George Efstathiou for his important comments and suggestions on the manuscript. D.K.H and A.S wish to acknowledge support from the Korea Ministry of Education, Science and Technology, Gyeongsangbuk-Do and Pohang City for Independent Junior Research Groups at the Asia Pacific Center for Theoretical Physics. The authors would like to thank Simon Prunet for his help in understanding Planck covariance matrix. A.S. would like to acknowledge the support of the National Research Foundation of Korea (NRF-2013R1A1A2013795). We acknowledge the use of WMAP-9 data and likelihood from Legacy Archive for Microwave Background Data Analysis (LAMBDA) [22] and Planck data and likelihood from Planck Legacy Archive (PLA) [29]. We also acknowledge the use of publicly available CosmoMC in our analysis.

\section{References}

[1] See, http://www. sciops.esa.int/index.php?project=planck\&page=Planck_Collaboration 
[2] L. A. Kofman and D. Y. .Pogosian, Phys. Lett. B 214 (1988) 508; D. S. Salopek, J. R. Bond and J. M. Bardeen, Phys. Rev. D 40 (1989) 1753; A. A. Starobinsky, JETP Lett. 55 (1992) 489 [Pisma Zh. Eksp. Teor. Fiz. 55 (1992) 477]; D. Polarski and A. A. Starobinsky, Nucl. Phys. B 385 (1992) 623; J. A. Adams, G. G. Ross and S. Sarkar, Nucl. Phys. B 503 (1997) 405 [hep-ph/9704286]; D. J. H. Chung, E. W. Kolb, A. Riotto and I. I. Tkachev, Phys. Rev. D 62 (2000) 043508 [hep-ph/9910437]; J. Martin, A. Riazuelo and M. Sakellariadou, Phys. Rev. D 61 (2000) 083518 [astro-ph/9904167]; J. Martin and R. H. Brandenberger, Phys. Rev. D 63 (2001) 123501 [hep-th/0005209]; J. Barriga, E. Gaztanaga, M. G. Santos and S. Sarkar, Mon. Not. Roy. Astron. Soc. 324 (2001) 977 [astro-ph/0011398]; J. A. Adams, B. Cresswell and R. Easther, Phys. Rev. D 64 (2001) 123514 [astro-ph/0102236]; U. H. Danielsson, Phys. Rev. D 66 (2002) 023511 [hep-th/0203198]; C. R. Contaldi, M. Peloso, L. Kofman and A. D. Linde, JCAP 0307, 002 (2003) [astro-ph/0303636]; O. Elgaroy, S. Hannestad and T. Haugboelle, JCAP 0309, 008 (2003) [astro-ph/0306229]; N. Kaloper and M. Kaplinghat, Phys. Rev. D 68 (2003) 123522 [hep-th/0307016]; H. V. Peiris et al. [WMAP Collaboration], Astrophys. J. Suppl. 148 (2003) 213 [astro-ph/0302225]; J. Martin and C. Ringeval, Phys. Rev. D 69 (2004) 083515 [astro-ph/0310382]; J. Martin and C. Ringeval, Phys. Rev. D 69 (2004) 127303 [astro-ph/0402609]; J. Martin and C. Ringeval, JCAP 0501, 007 (2005) [hep-ph/0405249]; L. Sriramkumar and T. Padmanabhan, Phys. Rev. D 71, 103512 (2005); [gr-qc/0408034]. P. Hunt and S. Sarkar, Phys. Rev. D 70 (2004) 103518 [astro-ph/0408138]; M. Bridges, A. N. Lasenby and M. P. Hobson, Mon. Not. Roy. Astron. Soc. 369 (2006) 1123 [astro-ph/0511573]; R. Allahverdi, K. Enqvist, J. Garcia-Bellido and A. Mazumdar, Phys. Rev. Lett. 97 (2006) 191304 [hep-ph/0605035]; A. Ashoorioon and A. Krause, hep-th/0607001; R. Allahverdi, K. Enqvist, J. Garcia-Bellido, A. Jokinen and A. Mazumdar, JCAP 0706, 019 (2007) [hep-ph/0610134]; J. C. Bueno Sanchez, K. Dimopoulos and D. H. Lyth, JCAP 0701, 015 (2007) [hep-ph/0608299]; L. Covi, J. Hamann, A. Melchiorri, A. Slosar and I. Sorbera, Phys. Rev. D 74 (2006) 083509 [astro-ph/0606452]; J. M. Cline and L. Hoi, JCAP 0606, 007 (2006) [astro-ph/0603403]; D. N. Spergel et al. [WMAP Collaboration], Astrophys. J. Suppl. 170 (2007) 377 [astro-ph/0603449]; P. Hunt and S. Sarkar, Phys. Rev. D 76 (2007) 123504 [arXiv:0706.2443 [astro-ph]]; J. Hamann, L. Covi, A. Melchiorri and A. Slosar, Phys. Rev. D 76 (2007) 023503 [astro-ph/0701380]; M. Joy, V. Sahni and A. A. Starobinsky, Phys. Rev. D 77 (2008) 023514 [arXiv:0711.1585 [astro-ph]]; A. Ashoorioon, A. Krause and K. Turzynski, JCAP 0902, 014 (2009) [arXiv:0810.4660 [hep-th]]; M. Joy, A. Shafieloo, V. Sahni and A. A. Starobinsky, JCAP 0906, 028 (2009) [arXiv:0807.3334 [astro-ph]]; R. K. Jain, P. Chingangbam, J. -O. Gong, L. Sriramkumar and T. Souradeep, JCAP 0901, 009 (2009) [arXiv:0809.3915 [astro-ph]]; C. Pahud, M. Kamionkowski and A. RLiddle, Phys. Rev. D 79 (2009) 083503 [arXiv:0807.0322 [astro-ph]]; R. Flauger, L. McAllister, E. Pajer, A. Westphal and G. Xu, JCAP 1006, 009 (2010) [arXiv:0907.2916 [hep-th]]; M. J. Mortonson, C. Dvorkin, H. V. Peiris and W. Hu, Phys. Rev. D 79 (2009) 103519 [arXiv:0903.4920 [astro-ph.CO]]; R. K. Jain, P. Chingangbam, L. Sriramkumar and T. Souradeep, Phys. Rev. D 82 (2010) 023509 [arXiv:0904.2518 [astro-ph.CO]]; C. Dvorkin and W. Hu, Phys. Rev. D 81 (2010) 023518 [arXiv:0910.2237 [astro-ph.CO]]; N. Barnaby and Z. Huang, Phys. Rev. D 80 (2009) 126018 [arXiv:0909.0751 [astro-ph.CO]]; K. Ichiki, R. Nagata and J. 'i. Yokoyama, Phys. Rev. 
D 81 (2010) 083010 [arXiv:0911.5108 [astro-ph.CO]]; J. Hamann, A. Shafieloo and T. Souradeep, JCAP 1004, 010 (2010) [arXiv:0912.2728 [astro-ph.CO]]; H. V. Peiris and L. Verde, Phys. Rev. D 81 (2010) 021302 [arXiv:0912.0268 [astro-ph.CO]]; D. K. Hazra, M. Aich, R. K. Jain, L. Sriramkumar and T. Souradeep, JCAP 1010, 008 (2010) [arXiv:1005.2175 [astro-ph.CO]]; A. Achucarro, J. -O. Gong, S. Hardeman, G. A. Palma and S. P. Patil, JCAP 1101, 030 (2011) [arXiv:1010.3693 [hep-ph]]; W. Hu, Phys. Rev. D 84 (2011) 027303 [arXiv:1104.4500 [astro-ph.CO]]; M. Aich, D. K. Hazra, L. Sriramkumar and T. Souradeep, Phys. Rev. D 87, 083526 (2013) [arXiv:1106.2798 [astro-ph.CO]]; D. K. Hazra, JCAP 1303, 003 (2013) [arXiv:1210.7170 [astro-ph.CO]]; V. Miranda, W. Hu and P. Adshead, Phys. Rev. D 86, 063529 (2012) [arXiv:1207.2186 [astro-ph.CO]]; H. Peiris, R. Easther and R. Flauger, arXiv:1303.2616 [astro-ph.CO]; R. Easther and R. Flauger, arXiv:1308.3736 [astro-ph.CO]; M. Benetti, arXiv:1308.6406 [astro-ph.CO]; P. D. Meerburg, D. N. Spergel and B. D. Wandelt, arXiv:1308.3704 [astro-ph.CO]; V. Miranda and W. Hu, arXiv:1312.0946 [astro-ph.CO]; S. Avila, J. Martin and D. Steer, arXiv:1304.3262 [hep-th]; A. Achucarro, V. Atal, P. Ortiz and J. Torrado, arXiv:1311.2552 [astro-ph.CO]; R. de Putter, E. V. Linder and A. Mishra, arXiv:1401.7022 [astro-ph.CO]; W. J. Handley, S. D. Brechet, A. N. Lasenby and M. P. Hobson, arXiv:1401.2253 [astro-ph.CO]; P. Adshead and W. Hu, arXiv:1402.1677 [astro-ph.CO].

[3] D. K. Hazra, A. Shafieloo and T. Souradeep, JCAP 1307, 031 (2013) [arXiv:1303.4143 [astro-ph.CO]].

[4] G. Hinshaw, D. Larson, E. Komatsu, D. N. Spergel, C. L. Bennett, J. Dunkley, M. R. Nolta and M. Halpern et al., arXiv:1212.5226 [astro-ph.CO].

[5] P. A. R. Ade et al. [Planck Collaboration], arXiv:1303.5082 [astro-ph.CO].

[6] P. A. R. Ade et al. [Planck Collaboration], arXiv:1303.5077 [astro-ph.CO].

[7] D. K. Hazra, A. Shafieloo and T. Souradeep, Phys. Rev. D 87, 123528 (2013) [arXiv:1303.5336 [astro-ph.CO]].

[8] D. K. Hazra, A. Shafieloo and G. F. Smoot, JCAP 1312, 035 (2013) [arXiv:1310.3038 [astro-ph.CO]].

[9] A. Shafieloo and T. Souradeep, Phys. Rev. D 70 (2004) 043523 [astro-ph/0312174].

[10] A. Shafieloo and T. Souradeep, Phys. Rev. D 78 (2008) 023511 [arXiv:0709.1944 [astro-ph]].

[11] S. Hannestad, Phys. Rev. D 63 (2001) 043009 [astro-ph/0009296]; M. Tegmark and M. Zaldarriaga, Phys. Rev. D 66 (2002) 103508 [astro-ph/0207047]; S. L. Bridle, A. M. Lewis, J. Weller and G. Efstathiou, Mon. Not. Roy. Astron. Soc. 342 (2003) L72 [astro-ph/0302306]; P. Mukherjee and Y. Wang, Astrophys. J. 599 (2003) 1 [astro-ph/0303211]; S. Hannestad, JCAP 0404, 002 (2004) [astro-ph/0311491]; D. Tocchini-Valentini, Y. Hoffman and J. Silk, Mon. Not. Roy. Astron. Soc. 367 (2006) 1095 [astro-ph/0509478]; N. Kogo, M. Sasaki and J. 'i. Yokoyama, Prog. Theor. Phys. 114 (2005) 555 [astro-ph/0504471]; S. M. Leach, Mon. Not. Roy. Astron. Soc. 372 (2006) 646 [astro-ph/0506390]; R. Nagata and J. 'i. Yokoyama, Phys. Rev. D 78 (2008) 123002 [arXiv:0809.4537 [astro-ph]]; R. Nagata and J. 'i. Yokoyama, Phys. Rev. D 79 (2009) 043010 [arXiv:0812.4585 [astro-ph]]; P. Paykari and A. H. Jaffe, 
Astrophys. J. 711 (2010) 1 [arXiv:0902.4399 [astro-ph.CO]]; K. Ichiki and R. Nagata, Phys. Rev. D 80 (2009) 083002; A. Shafieloo and T. Souradeep, New J. Phys. 13 (2011) 103024 [arXiv:0901.0716 [astro-ph.CO]]; G. Nicholson and C. R. Contaldi, JCAP 0907, 011 (2009) [arXiv:0903.1106 [astro-ph.CO]]; G. Nicholson, C. R. Contaldi and P. Paykari, JCAP 1001, 016 (2010) [arXiv:0909.5092 [astro-ph.CO]]; M. Bridges et al, Mon. Not. R. Astron. Soc. 400 1075B (2009); C. Gauthier and M. Bucher, JCAP 1210, 050 (2012) [arXiv:1209.2147 [astro-ph.CO]]; R. Hlozek, J. Dunkley, G. Addison, J. W. Appel, J. R. Bond, C. S. Carvalho, S. Das and M. Devlin et al., Astrophys. J. 749 (2012) 90 [arXiv:1105.4887 [astro-ph.CO]]; J. A. Vazquez, M. Bridges, M. P. Hobson and A. N. Lasenby, JCAP 1206, 006 (2012) [arXiv:1203.1252 [astro-ph.CO]]; G. Goswami and J. Prasad, Phys. Rev. D 88, 023522 (2013) [arXiv:1303.4747 [astro-ph.CO]]; P. Hunt and S. Sarkar, arXiv:1308.2317 [astro-ph.CO].

[12] P. Paykari, F. Lanusse, J.-L. Starck, F. Sureau and J. Bobin, arXiv:1406.7725 [astro-ph.CO].

[13] B. H. Richardson, J. Opt. Soc. Am. 6255 (1972)

[14] L. B. Lucy, Astron. J. 796 (1974)

[15] C. M. Baugh and G. Efstathiou, Mon. Not. R. Astron. Soc. 265145 (1993)

[16] C. M. Baugh and G. Efstathiou, Mon. Not. R. Astron. Soc. 267323 (1994)

[17] P. A. R. Ade et al. [Planck Collaboration], arXiv:1303.5076 [astro-ph.CO].

[18] P. A. R. Ade et al. [Planck Collaboration], arXiv:1303.5075 [astro-ph.CO].

[19] See, http://www.sciops.esa.int/wikiSI/planckpla/index.php?title= CMB_spectrum_\%26_Likelihood_Code\&instance=Planck_Public_PLA

[20] See, http://camb.info/.

[21] A. Lewis, A. Challinor and A. Lasenby, Astrophys. J. 538 (2000) 473 [astro-ph/9911177].

[22] See, http://lambda.gsfc.nasa.gov/product/map/dr3/m_products.cfm

[23] D. K. Hazra and A. Shafieloo, Phys. Rev. D 89, 043004 (2014) [arXiv:1308.2911 [astro-ph.CO]].

[24] A. sKovcs, J. Carron and I. nSzapudi, arXiv:1307.1111 [astro-ph.CO].

[25] D. K. Hazra and A. Shafieloo, work in progress.

[26] D. K. Hazra and A. Shafieloo, JCAP 1401, 043 (2014) [arXiv:1401.0595 [astro-ph.CO]].

[27] A. Shafieloo, T. Souradeep, P. Manimaran, P. K. Panigrahi and R. Rangarajan, Phys. Rev. D 75 (2007) 123502 [astro-ph/0611352].

[28] A. Shafieloo, Mon. Not. Roy. Astron. Soc. 380, 1573 (2007) [astro-ph/0703034 [ASTRO-PH]].

[29] http://www. sciops .esa $\cdot$ int/index $\cdot$ php?project=planck\&page= Planck_Legacy_Archive 